\title{
Quantum Heisenberg antiferromagnets: a survey of the activity in Firenze
}

\author{
Umberto Balucani, ${ }^{1}$ Luca Capriotti, ${ }^{2,3}$ Alessandro Cuccoli, ${ }^{4,5}$ Andrea Fubini, ${ }^{4,5}$ \\ Tommaso Roscilde, ${ }^{6}$ Valerio Tognetti, ${ }^{4,5}$ Ruggero Vaia, ${ }^{1,5}$ and Paola Verrucchi ${ }^{5,1}$ \\ ${ }^{1}$ Istituto dei Sistemi Complessi, Consiglio Nazionale delle Ricerche, \\ via Madonna del Piano, I-50019 Sesto Fiorentino (FI), Italy \\ ${ }^{2}$ Valuation Risk Group, Credit Suisse First Boston (Europe) Ltd., \\ One Cabot Square, London E14 4QJ, United Kingdom \\ ${ }^{3}$ Kavli Institute for Theoretical Physics, University of California, Santa Barbara, CA 93106, USA \\ ${ }^{4}$ Dipartimento di Fisica dell'Università di Firenze, \\ via G. Sansone 1, I-50019 Sesto Fiorentino (FI), Italy \\ ${ }^{5}$ Istituto Nazionale per la Fisica della Materia, Unità di Ricerca di Firenze, \\ via G. Sansone 1, I-50019 Sesto Fiorentino (FI), Italy \\ ${ }^{6}$ Department of Physics and Astronomy, University of Southern California, Los Angeles, CA 90089-0484, USA
}

(Dated: September 11, 2018)

\begin{abstract}
Over the years the research group in Firenze has produced a number of theoretical results concerning the statistical mechanics of quantum antiferromagnetic models, which range from the theory of two-magnon Raman scattering to the characterization of the phase transitions in quantum low-dimensional antiferromagnetic models. Our research activity was steadily aimed to the understanding of experimental observations.
\end{abstract}

\section{INTRODUCTION}

The Heisenberg model may well be considered the cornerstone of the modern theory of magnetic systems; the reason for such an important role is the simple structure of the Hamiltonian, whose symmetries underlie its peculiar features. The basic forces which determine the alignment of the spins, are represented by the exchange integrals J's. At variance with the ferromagnet where the parallel alignment is promoted, in the antiferromagnet a lot of peculiar arrangements of the spins can occur, with strong differences between classical and quantum systems. As matter of fact, also for nearest-neighbor antiferromagnetic interactions the ground state of the Hamiltonian is different from the Néel state with antialigned spins, and the (staggered) magnetization shows the so called spin reduction with respect to the saturation value also at $T=0$. The linear excitations of an antiferromagnet can be roughly associated in two families and pair excitations with vanishing total magnetization are possible: the fact that the total momentum of these can be close to zero allows for their investigation by light scattering.

While these peculiar features of antiferromagnetism already occur in three-dimensional (3D) compounds, they are more pronounced in the low-dimensional ones, where other effects caused by the enhanced role of classical and quantum fluctuations are present and exotic spin configurations associated with field theory models can appear. Indeed, the last two decades have seen a renewed interest both in the case of the one-dimensional (1D) quantum Heisenberg antiferromagnet (QHAF), for which a peculiar behavior of the ground state vs spin value was predicted $^{1}$, and of the two-dimensional (2D) QHAF, because of its theoretically challenging properties and of the fact that it models the magnetic behavior of the parent compounds of some high- $T_{\mathrm{c}}$ superconductors ${ }^{2.3}$. The experimental activity on $2 \mathrm{D}$ antiferromagnets stems from the existence of several real compounds whose crystal structure is such that the magnetic ions form parallel planes and interact strongly only if belonging to the same plane. As a consequence of such structure, their magnetic behavior is indeed 2D down to those low temperatures where the weak interplane interaction becomes relevant, driving the system towards a 3D ordered phase.

In addition, the 2D Heisenberg model can be enriched trough symmetry-breaking terms - we considered easyaxis (EA) and easy-plane (EP) anisotropy, as well as an external uniform magnetic field - which are useful to reproduce the experimental behavior of many layered compounds. In the EA case one is left with a discrete reflection symmetry and the system undergoes an Isinglike phase transition. In the EP case or when a magnetic field is applied the residual $O(2)$ symmetry prevents finite-temperature ordering ${ }^{4}$, but vortex excitations are possible and determine a Berezinskii-Kosterlitz-Thouless (BKT) transition between a paramagnetic and a quasiordered phase. In spite of the tiny anisotropies of real systems (usually $\lesssim 0.01 \mathrm{~J}$ ), it can be shown that they dramatically change the behavior of the spin array already at temperatures of the order of $J$.

In this paper we report about the progresses in the theory of Heisenberg antiferromagnets that have been obtained by our group in Firenze. The early work on the theory of two-magnon Raman scattering is summarized in Section III while the following Sections report about the recent activity on low-dimensional antiferromagnetism. Section III is devoted to 1D models, and concerns the study of the effect of soliton-like excitations in the compound TMMC, as well as the anisotropic spin1 model, for which a reduced description of the ground 
state allows one to investigate the quantum phase transition in a unitarily transformed representation and to obtain quantitative results for the phase diagram. Section IV concerns the theory of the isotropic 2D QHAF, for which we reproduced the experimental correlation length by means of a semiclassical approach, also deriving the connection with (and the limitations of) famous quantum field theory results. In Section $\nabla$ we summarize several recent results concerning the anisotropic 2D QHAF, with emphasis onto the different phase diagrams and the experimentally measurable signatures of XY or Ising behavior. Eventually, in Section $\nabla]$ results on the 2D frustrated $J_{1}-J_{2}$ isotropic model are described.

\section{TWO-MAGNON RAMAN SCATTERING IN HEISENBERG ANTIFERROMAGNETS}

The scattering of radiation is a very powerful tool to study elementary excitations in Condensed Matter Physics. Any complete experiment gives rise to a quasielastic component due to non-propagating or diffusive modes and to symmetrically shifted spectra corresponding to the states of the system under investigation with an amplitude ratio governed by the detailed balance principle. The most sensitive probes for this investigation are undoubtedly thermal neutrons, because the characteristic energies and wavevectors fit very well with those of the magnetic elementary excitations. However, light-scattering experiments can require a simpler apparatus and offer a better accuracy, although the transfer wavevector $\boldsymbol{k}$ is much smaller than the size of the Brillouin zone so that usually only the center of this zone can be directly probed. In spite of this, two-spin Raman scattering involving the creation and destruction of a pair of elementary excitations can be performed, with the contribution of two magnons having equal frequencies and opposite wavevectors. This two-magnon scattering is expected to be spread over a band of frequencies in antiferromagnets. However, the density of states strongly enhances the contribution of zone-boundary (ZB) excitations ${ }^{\underline{5}}$, i.e., at $\boldsymbol{k} \sim \boldsymbol{k}_{\mathrm{ZB}}$.

While in ferromagnets the two-spin process is only due to a second order mechanism, orders of magnitude smaller than the first order one, in antiferromagnets a different independent process is permitted, stronger than the corresponding one for single-spin spectra ${ }^{6}$. Specifically, an exchange mechanism does not change the total $z$-component of the spins: exciting two magnons in the two different sublattices $(\Delta M=0)^{\underline{\underline{Z}}}$ is the dominant scattering process.

The one-spin Raman scattering peak disappears at the Néel temperature because it probes the smallest wavevectors, related with the long-range correlations. In contrast, two-magnon Raman scattering essentially probes the highest wavevectors, related to short-range correlations. Therefore two-spin Raman scattering features persist also in the paramagnetic phase $e^{\frac{7}{}}$ where short-range order is still present.

Let us consider the following antiferromagnetic Hamiltonian with exchange integral $J>0, z$ nearest neighbors with displacements labeled by $\boldsymbol{d}$, and two $(a, b)$ sublattices ${ }^{8}$ :

$$
\mathcal{H}=\frac{J}{2} \sum_{i \boldsymbol{d}} \boldsymbol{S}_{\boldsymbol{i}, a} \cdot \boldsymbol{S}_{\boldsymbol{i}+\boldsymbol{d}, b}
$$

The scattering cross section $\mathcal{S}(\omega)$ turns out ${ }^{9}$ to be proportional to the Fourier transform of $\langle M(0) M(t)\rangle$, where

$$
M=\sum_{k} \mathcal{M}_{k} \boldsymbol{S}_{k} \cdot \boldsymbol{S}_{-k},
$$

is the effective Raman scattering operator.

Many antiferromagnetic compounds can be mapped onto this model, even though a small next-nearestneighbor exchange interaction without competitive effects, as well as anisotropy terms could be present. For instance, there are $3 \mathrm{D}$ perovskite and rutile structures (e.g., $\mathrm{KNiF}_{4}, \mathrm{NiF}_{2}$ ) and $2 \mathrm{D}$ layered structures (e.g., $\mathrm{K}_{2} \mathrm{NiF}_{4}$, $\mathrm{LaCuO}_{2}$ ).

Let us remember that the exact ground state is not exactly known, except in 1D models with $S=1 / 2$ or $S=\infty$ (i.e., the classical case): in the latter case it coincides with the 'Néel state' with antialigned sublattices.

In the ordered phase the theory can be developed in terms of two families of magnon operators $\left(\alpha_{\boldsymbol{k}}, \beta_{\boldsymbol{k}}\right)$, through the Dyson-Maleev spin-boson transformation and a Bogoliubov transformation:

$$
\mathcal{H}=E_{0}+\mathcal{H}_{0}+V,
$$

where $E_{0}$ is the ground state energy in interacting spinwave approximation and

$$
\mathcal{H}_{0}=\sum_{\boldsymbol{k}} \omega_{\boldsymbol{k}}\left(\alpha_{\boldsymbol{k}}^{\dagger} \alpha_{\boldsymbol{k}}+\beta_{\boldsymbol{k}}^{\dagger} \beta_{\boldsymbol{k}}\right)
$$

is the quadratic part of the Hamiltonian of a magnon gas whose frequencies, renormalized by zero- $T$ quantum fluctuations, are

$$
\omega_{\boldsymbol{k}}=J S z\left(1+\frac{C}{2 S}\right) \sqrt{1-\gamma_{\boldsymbol{k}}^{2}} ; \quad \gamma_{\boldsymbol{k}}=\frac{1}{z} \sum_{\boldsymbol{d}} e^{-i \boldsymbol{k} \cdot \boldsymbol{d}} .
$$

The last term in the Hamiltonian, $V$, represents the four-magnon interaction, whose most significant term refers to two magnons of each family and turns out to be:

$$
V=2 \frac{J z}{N} \sum_{\boldsymbol{q} \boldsymbol{q}^{\prime} \boldsymbol{p} \boldsymbol{p}^{\prime}} \delta_{\boldsymbol{q}+\boldsymbol{p}, \boldsymbol{q}^{\prime}+\boldsymbol{p}^{\prime}} I_{\boldsymbol{q} \boldsymbol{q}^{\prime}, \boldsymbol{p} \boldsymbol{p}^{\prime}}^{\alpha \beta} \alpha_{\boldsymbol{q}}^{\dagger} \alpha_{\boldsymbol{q}^{\prime}} \beta_{\boldsymbol{p}}^{\dagger} \beta_{\boldsymbol{p}^{\prime}},
$$

where the coefficients $I_{\boldsymbol{q} \boldsymbol{q}^{\prime}, \boldsymbol{p} \boldsymbol{p}^{\prime}}^{\alpha \beta}$ are known functions of $\gamma_{\boldsymbol{k}}$.

In the Hartree-Fock approximation ${ }^{10}$ the temperature dependent Raman scattering operator (2) can be written

$$
M=\alpha(T) S \sum_{\boldsymbol{k}} \Phi_{\boldsymbol{k}}\left(\alpha_{\boldsymbol{k}} \beta_{\boldsymbol{k}}+\alpha_{\boldsymbol{k}}^{\dagger} \beta_{\boldsymbol{k}}^{\dagger}\right)
$$




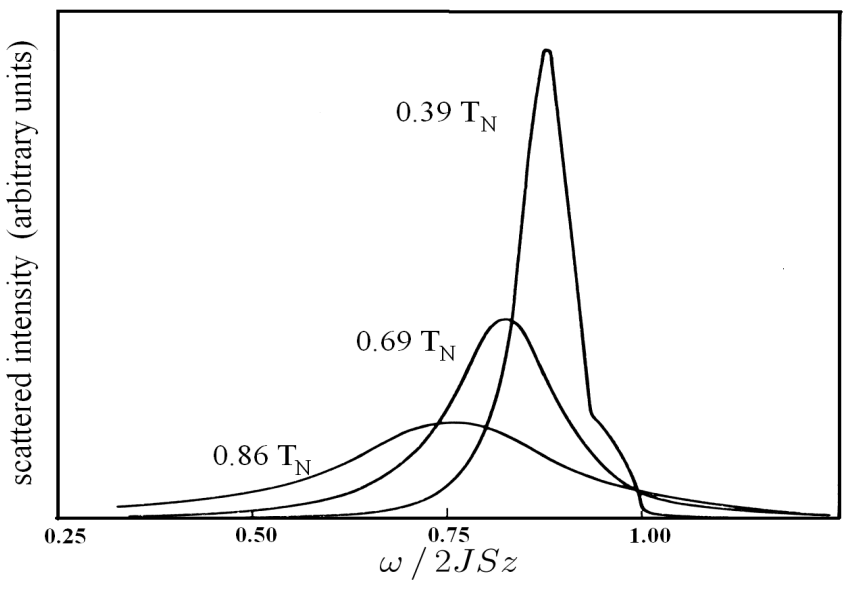

FIG. 1: Theoretical two-magnon spectra in $\mathrm{KNiF}_{3}$ at different temperatures ${ }^{10}$.

with $\omega_{\boldsymbol{k}}(T)=\alpha(T) \omega_{\boldsymbol{k}}$. The two magnons created or destroyed by the operator (7) interact through $V$ as given by (6), so that the peak of the cross section $\mathcal{S}(\omega)$ appears at values smaller that $2 \omega_{\mathrm{zB}}$ for an amount of the order of $J$. The explicit $\mathcal{S}(\omega)$ at $T=0$ was calculated in the 'ladder approximation' by Elliott and Thorpe and found in very good agreement with experiments 9 .

The finite temperature calculation of the two-magnon Raman scattering cross section in the ordered region, up to $T \sim 0.95 T_{\mathrm{N}}$ was performed by Balucani and Tognetti10, calculating the two-magnon propagator in the 'ladder approximation', taking also into account the damping and the temperature renormalization of the magnons at the boundary of the Brillouin zone ${ }^{11}$. The calculated spectra $\mathcal{S}(\omega)$, at increasing temperatures, were found in very good agreement with the experimental ones 8 and their characteristic parameters (peak and linewidth) permitted to determine the temperature behavior of the frequency and damping of the $\mathrm{ZB}$ magnons ${ }^{12}$. In Figs. 1 and 2 we show the excellent agreement of our theoretical approach with the experiments in the ordered phase ${ }^{13}$. The validity of light scattering in probing the characteristic of ZB magnons has been confirmed both from the theoretical and the experimental point of view $^{12}$. In Fig. 3] our theoretical ZB magnon damping calculations are compared with experimental data from different techniques.

In the paramagnetic phase all experimental spectra show the persistence of a broad inelastic peak up to $T \sim 1.4 T_{N}$. Only at $T \gg T_{N}$ the spectra have a structureless shape centered around $\omega=0$. As matter of fact, the highest wavevectors sample only the behavior of clusters of neighboring spins, thus giving a measure of the short-range antiferromagnetic order that is present at all finite temperatures.

In the disordered phase conventional many-body methods are of little use for a quantitative interpretation of the observed largely spread spectra. The concept of quasiparticle loses its meaning because of the overdamped

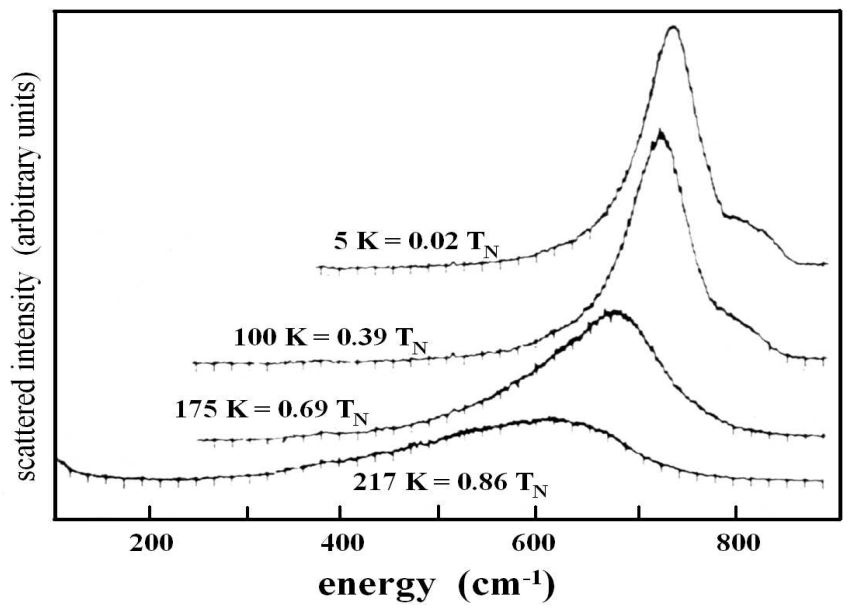

FIG. 2: Experimental two-magnon spectra in $\mathrm{KNiF}_{3}$ at different temperatures ${ }^{5}$.

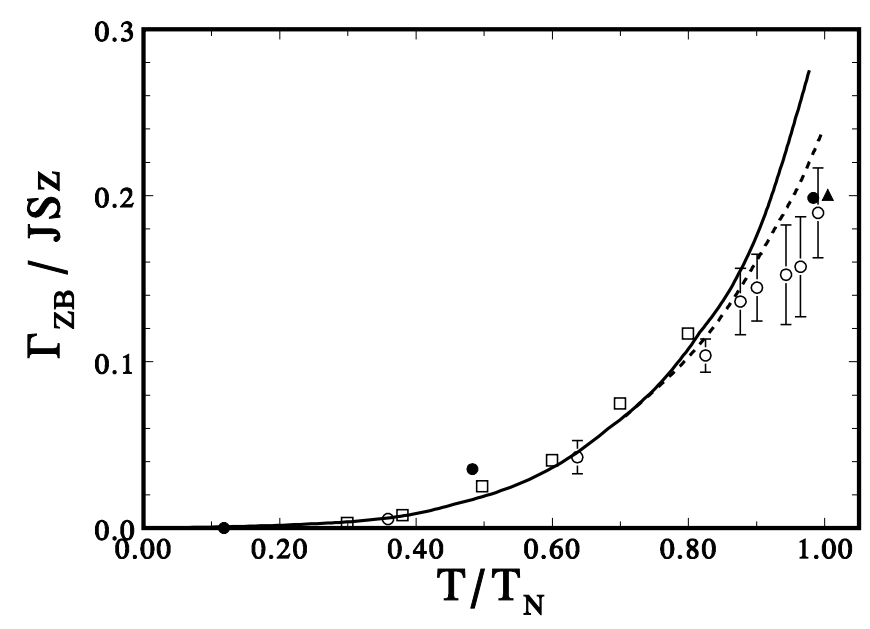

FIG. 3: Zone-boundary damping $\Gamma_{\mathrm{ZB}}$ vs temperature. The symbols refer to different experimental approaches: in particular the open circles are our light scattering data 12 . The dashed line is an improvement ${ }^{12}$ to a previous (solid) theoretical curve ${ }^{10}$.

character of the 'excitations'. The calculation of $\mathcal{S}(\omega)$ can be instead approached by other more general theoretical methods devoted to the representation of the dynamical correlation functions based on the linear response theory ${ }^{11}$. Let us consider the 'Kubo relaxation function' associated with our scattering process:

$$
f_{0}(t) \equiv \frac{1}{\langle M(0) M(0)\rangle} \int_{0}^{\beta} d \lambda\left\langle e^{\lambda \mathcal{H}} M(0) e^{-\lambda \mathcal{H}} M(t)\right\rangle .
$$

Its Laplace transform $f_{0}(z)$ is related to the scattering cross section:

$$
\mathcal{S}(\omega) \propto \frac{\omega}{1-e^{-\beta \omega}} \Re f_{0}(z=i \omega) .
$$

Mori ${ }^{14}$ has given the following continued fraction repre- 


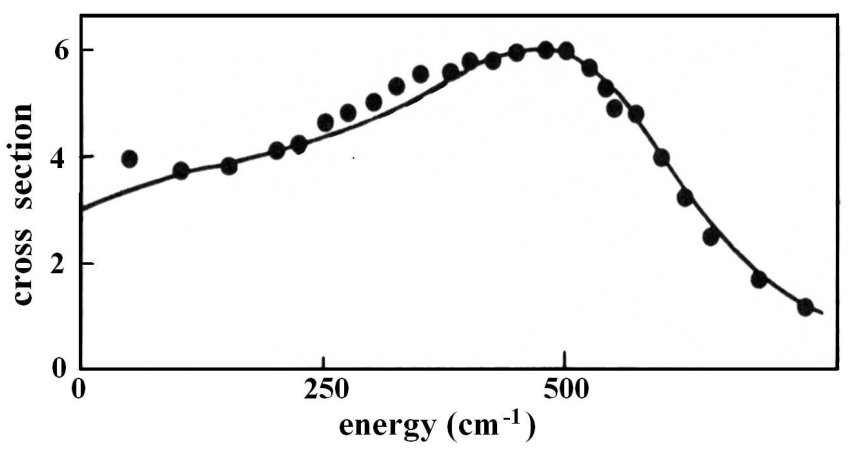

FIG. 4: Two-spin Stokes spectrum in $\mathrm{KNiF}_{3}$ at $T \simeq 1.02 T_{\mathrm{N}}$. The line reports the theoretical shape ${ }^{15}$, compared with experimental data.

sentation of the relaxation function 11 :

$$
f_{0}(z)=\frac{1}{z+\Delta_{1} f_{1}(z)} ; \quad f_{n}(z)=\frac{1}{z+\Delta_{n+1} f_{n+1}(z)},
$$

which is formally exact, but allow us to do some approximations about the level of the termination $f_{n+1}(z)$. The quantities $\Delta_{n}$ can be expressed in terms of frequency moments:

$$
\left\langle\omega^{2 n}\right\rangle=\int_{-\infty}^{\infty} d \omega \omega^{2 n} f_{0}(\omega)
$$

In our calculations of $f_{0}(z)$ in the entire paramagnetic phase ${ }^{15.16}$, the coefficients $\Delta_{1}$ e $\Delta_{2}$ have been approximately evaluated by means of a decoupling procedure. Moreover, the third stage of the continued fraction (10) is evaluated assuming that

$$
\Delta_{3} f_{3}(z) \sim \Delta_{3}\left[f_{3}(0)+z f_{3}^{\prime}(0)\right] .
$$

The parameters involved in (12) can be estimated by the knowledge of the short time behavior of $f_{0}(t)$ determined by the first moments, $\left\langle\omega^{2}\right\rangle$ and $\left\langle\omega^{4}\right\rangle$.

The results of our approach in the paramagnetic region are compared with the experiment in Fig. [4 showing the persistence of the peak of the ZB magnetic excitations above the critical temperature.

\section{THE ONE-DIMENSIONAL ANTIFERROMAGNET}

\section{A. Solitons in the antiferromagnet TMMC}

Interest in low-dimensional systems is motivated by the much greater simplicity of calculation as compared with the $3 \mathrm{D}$ ones. The powerful mathematical approach based on the inverse-scattering and Bethe Ansatz techniques permits to exactly solve some 1D models, calculating thermodynamic and sometimes transport quantities both in classical and quantum cases $\frac{17}{}$. The most celebrated realizations of these models occur in 1D magnets. An original suggestion by Mikesk $\alpha^{18}$ was that the antiferromagnetic chain TMMC $\left[\left(\mathrm{CH}_{3}\right)_{4} \mathrm{NMnCl}_{3}\right]$ can be mapped onto a sine-Gordon classical 1D model. The elementary excitations of the sine-Gordon field are given in terms of linear small-amplitude spin-waves and nonlinear breathers and kink-solitons. The non-linear elementary excitations give a detectable contribution to the magnetic specific heat.

TMMC is composed of Heisenberg $(S=5 / 2)$ antiferromagnetic chains along the $z$-axis:

$$
\mathcal{H}=J \sum_{i}\left(\boldsymbol{S}_{i} \cdot \boldsymbol{S}_{i+1}-\delta S_{i}^{z} S_{i+1}^{z}\right)
$$

with a very small easy-plane anisotropy $(\delta=0.0086)$.

A magnetic field of the order of $1 \div 10 \mathrm{~T}$ can be applied perpendicularly ( $y$-axis) or along the chain . In the first case, with approximations the more valid the lower the magnetic field $(H<5 \mathrm{~T})$, in the continuum limit TMMC can be represented by the classical sine-Gordon Hamiltonian:

$$
\mathcal{H}=\frac{A}{2} \int d x\left[\dot{\Phi}^{2}+c_{0}^{2} \Phi_{x}^{2}+2 \omega_{0}^{2}(1-\cos \Phi)\right],
$$

whose parameters are related with the magnetic Hamiltonian (13), the reduced magnetic field $h=g \mu_{\mathrm{B}} H$, and the lattice spacing $a$ as follows:

$$
A=\frac{1}{8 J a}, \quad c_{0}=a J S \sqrt{1-\frac{\delta}{2}}, \quad \omega_{0}=h \sqrt{1-\frac{\delta}{2}} .
$$

The energy of kink-soliton turns out to be

$$
E_{s}=8 A \omega_{0} c_{0} \simeq h S
$$

and depends on the applied field. At difference with the ferromagnetic solitons, these solitons can be easily excited at lowest temperatures and can give a significant contribution to the thermodynamics $\frac{19}{9}$. When the field is applied longitudinally along the $z$-axis only spin-waves are present: therefore, the specific-heat measurements were performed in the two configurations. The contribution from the nonlinear excitations was obtained as the difference $\Delta C$ between the two experiments.

The thermodynamic quantities were calculated by the classical transfer-matrix method ${ }^{20}$ for the sine-Gordon model (14). We then used a classical discrete planar model21:

$$
\mathcal{H}=\sum_{i}\left[2 J S^{2} \cos \left(\Phi_{i}-\Phi_{i+1}\right)+h S\left(1-\cos \Phi_{i}\right)\right],
$$

verifying that it is qualitatively similar to the sineGordon. The comparison ${ }^{21}$ is shown in Fig. [5 where the linear spin-wave specific heat was subtracted to emphasize the nonlinear contribution, together with the prediction of the "classical soliton gas phenomenology'19. 


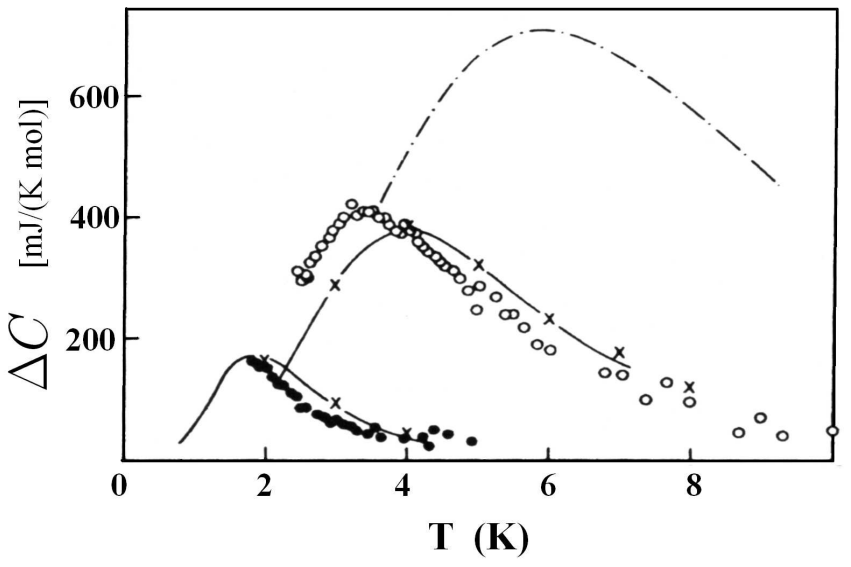

FIG. 5: Experimental contribution of nonlinear excitations to the specific heat of TMMC, $\Delta C=C(H)-C(0)-\Delta C$ SW . The field values are $H=5.39 \mathrm{~T}(\bullet)$ and $H=2.5 \mathrm{~T}(\circ)$. The dash-dotted line reports the result of the free soliton gas phenomenology. The planar model (interpolated crosses) appears to quantitatively explain the behavior of TMMC.

This proved the presence of nonlinear excitations similar to sine-Gordon solitons, but the peak of the specific heat occurs at temperatures where solitons cannot be considered to be non interacting and the 'classical soliton-gas phenomenology' breaks down. When the magnetic field is increased up to $9.98 \mathrm{~T}$ the model (17) is no more able to describe the experiments. A quasiuniaxial model 21 was proposed and found in good agreement. For general reference on the subject see 22 .

\section{B. The $S=1$ quantum antiferromagnet}

We here deal with quantum antiferromagnetic spin chains, focusing our attention on the class of models defined by the Hamiltonian

$$
\frac{\mathcal{H}}{J}=\sum_{i}\left[\left(S_{i}^{x} S_{i+1}^{x}+S_{i}^{y} S_{i+1}^{y}\right)+\lambda S_{i}^{z} S_{i+1}^{z}+d\left(S_{i}^{z}\right)^{2}\right]
$$

with exchange integral $J>0$ and single-ion anisotropy $d$.

One of the most surprising evidence of the difference between ferro- and antiferromagnetic systems is related with the so-called Haldane conjecture, i.e. with $T=0$ properties of integer-spin antiferromagnetic chains. In general, we expect three possible situations for the ground state of a magnetic system: either it is ordered (with finitely constant correlation functions), or quasi-ordered (with power-law decaying correlation functions), or completely disordered (with exponentially decaying correlation functions). One could intuitively expect the third option to be possibly dismissed, based on the idea that, when thermal fluctuations are completely suppressed, the system be in an ordered or at least quasiordered ground state. This idea is in fact proved correct for half-integer spin systems, thanks to the so called
Lieb-Schultz-Mattis theorem ${ }^{23}$. Despite the generalization of such theorem to integer-spin systems being impossible, its general validity has been taken for granted till 1983, when Haldane $\underline{\underline{1}}$ suggested, for the integerspin Heisenberg chain, an unexpected $T=0$ behavior: a unique and genuinely disordered ground state, meaning exponentially decaying correlation functions and a finite gap in the excitation spectrum. After more than two decades Haldane's idea that integer-spin systems can have a genuinely disordered ground state still stands as a conjecture. However, theoretical 24.25 .26 .27 , experimen-

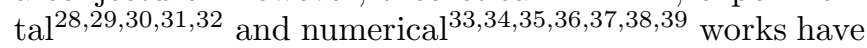
definitely confirmed its validity.

Let us consider Eq. (18) for integer spin: in the $(d, \lambda)$ plane one may identify different quantum phases, corresponding to models whose ground states share common features. For $\lambda>0$ three phases are singled out: the Néel phase $(\lambda \gg d)$, where the ground state has a Néellike structure, the so-called large- $d$ phase $(d \gg \lambda)$, where the ground state is characterized by a large majority of sites where $S^{z}=0$, and the Haldane phase, which extends around the isotropic point $(d=0, \lambda=1)$, and is characterized by disordered ground states.

We first deal with the Ising limit, $\mathcal{H} / J=\lambda \sum_{i} S_{i}^{z} S_{i+1}^{z}$ : Upon its ground state, the antiferromagnetically ordered Néel state, one may construct three types of excitations: a single deviation, a direct soliton, an indirect soliton, where direct (indirect) refers to the fact that the excitation be generated by flipping all the spins on the right of a given site while keeping the $z$ component of the spin on such site unchanged (setting it to zero). The above configurations have all energy $+2 \lambda$ with respect to that of the ground state, and do generate, when properly combined, all the excited states; amongst them, we concentrate upon those containing a couple of adjacent indirect solitons and notice that their energy is $+3 \lambda$, while excited states containing two separate indirect solitons have energy $+4 \lambda$. Therefore, indirect solitons are characterized by a bounding energy $\lambda$; moreover, one may easily see that isolated solitons may effectively introduce disorder in the global configuration of the system, while coupled solitons do only reduce the magnetization of each of the two antiferromagnetic sublattices ${ }^{40}$. In fact, strings containing any odd (even) number of adjacent solitons act on the order of the global configuration as if they were isolated (coupled) solitons.

As we move from the Ising limit, the transverse interaction $\sum_{i}\left(S_{i}^{x} S_{i+1}^{x}+S_{i}^{y} S_{i+1}^{y}\right)$ comes into play, and is seen ${ }^{41}$ to more efficiently lower the energy of the system by delocalizing indirect solitons rather than single deviations or direct solitons, thus indicating configurations which uniquely contain indirect solitons as crucial in understanding how the system evolves from the Ising limit (Néel phase) to the isotropic case(Haldane phase).

From the above ideas we may draw a simple but suggestive scheme for such evolution:

- in the Ising limit $(\lambda \rightarrow \infty)$ the ground state is the antiferromagnetically ordered Néel state; 
- as $\lambda$ decreases, indirect solitons appear along the chain in pairs, thus keeping the antiferromagnetic order;

- as $\lambda$ is further lowered, indirect soliton pairs dissociate due to the transverse interaction which, by spreading solitons along the chain, can cause the ground state to be disordered.

Due to the privileged role of indirect solitons in the above scheme, we concentrate on configurations which do only contain indirect solitons. Such configurations generate a subspace for the Hilbert space of the system, which is referred to, in the literature, as the reduced Hilbert space ${ }^{42}$. States belonging to the reduced Hilbert space are strongly characterized by the fact that if one eliminates all sites with $S^{z}=0$, a perfectly antiferromagnetically ordered chain is left. Remarkably, this type of order, which is called hidden order in the literature, is not destroyed by soliton pairs dissociation, and it actually characterizes the disordered ground state of a Haldane system, as discussed below.

In 1992 Kennedy and Tasaki (KT) defined a non-local unitary transformation ${ }^{43}$ which makes the hidden order visible, meanwhile clarifying its meaning. The transformation is defined by $U=(-1)^{N_{0}+[N / 2]} \prod_{k} U_{k}$ with

$$
U_{k}=\frac{1}{2}\left(e^{i \pi \sum_{p=1}^{k-1} S_{p}^{z}}-1\right) e^{i \pi S_{k}^{x}}+\frac{1}{2}\left(e^{i \pi \sum_{p=1}^{k-1} S_{p}^{z}}+1\right),
$$

where $N$ is the number of sites of the chain, $[N / 2]$ is the integer part of $N / 2$, and $N_{0}$ is the number of odd sites where $S^{z}=0$. If the pure state $|\Psi\rangle$ has hidden order, meaning that it only contains indirect solitons, then $U|\Psi\rangle$ has spins with $S^{z} \neq 0$ all parallel to each other. This point is made transparent by the introduction of the string order parameter ${ }^{44}$

$$
O_{\text {string }}^{\alpha}(\mathcal{H}) \equiv \lim _{|i-j| \rightarrow \infty}\left\langle S_{i}^{\alpha} \exp \left[i \pi \sum_{l=i}^{j-1} S_{l}^{\alpha}\right] S_{j}^{\alpha}\right\rangle_{\mathcal{H}},
$$

where $\alpha=x, y, z$, and $\langle\cdots\rangle_{\mathcal{H}}$ indicates the expectation value over the ground state of the Hamiltonian $\mathcal{H}$. It may be shown that $O_{\text {string }}^{z}(\mathcal{H}) \neq 0$ if and only if the ground state belongs to the reduced Hilbert space. In other terms, while ferromagnetic order is revealed by the ferromagnetic order parameter $O_{\text {ferro }}^{\alpha} \equiv \lim _{|i-j| \rightarrow \infty}\left\langle S_{i}^{\alpha} S_{j}^{\alpha}\right\rangle_{\mathcal{H}}$, the hidden order is revealed by the string order parameter Eq. (19). In fact, the non local transformation $U$ relates the above order parameters through the relation

$$
O_{\text {string }}^{\eta}=O_{\text {ferro }}^{\eta}\left(U \mathcal{H} U^{-1}\right)
$$

for $\eta=x, z$, meaning that the analysis of the hidden order in a system described by $\mathcal{H}$ may be developed by studying the ferromagnetic order in the system described by the transformed Hamiltonian $\widetilde{\mathcal{H}} \equiv U \mathcal{H} U^{-1}$, which reads, for $\mathcal{H}$ defined by Eq. (18),

$$
\frac{\widetilde{\mathcal{H}}}{J}=\sum_{i}\left[-S_{i}^{x} S_{i+1}^{x}+S_{i}^{y} e^{S_{i}^{z}+S_{i+1}^{x}} S_{i+1}^{y}-\lambda S_{i}^{z} S_{i+1}^{z}+d\left(S_{i}^{z}\right)^{2}\right] .
$$

Our work developed as follows: one first assumes that the relevant configurations, as far as the Néel-Haldane transition is concerned, belong to the reduced Hilbert space; this permits, by the KT transformation, to restrict the analysis to the subspace of states with either $S_{i}^{z}=1$ or $S_{i}^{z}=0, \forall i$. Then the expectation value of the transformed Hamiltonian $\widetilde{\mathcal{H}}$ Eq.(21) is minimized on a trial ground state whose structure takes into account at least short-range correlations between spins. By this procedure, we aim at following the effective dissociation of soliton pairs, in order to clarify the connection between the occurrence of isolated solitons in the ground state, and the transition towards the completely disordered Haldane-phase 39.41 .42 .45 .

In the framework of a standard variational approach, we should minimize $\left\langle\Phi_{0}|\mathcal{H}| \Phi_{0}\right\rangle$ with respect to a certain number of variational parameters entering the expression of the normalized trial ground-state $\left|\Phi_{0}\right\rangle$. By applying the non-local unitary transformation $\mathcal{U}$ we instead minimize $\left\langle\Psi_{0}\left|\mathcal{U H \mathcal { U }}^{-1}\right| \Psi_{0}\right\rangle$ with $\left|\Psi_{0}\right\rangle \equiv \mathcal{U}\left|\Phi_{0}\right\rangle$. and the transformed hamiltonian $\widetilde{\mathcal{H}} \equiv \mathcal{U} \mathcal{H U}^{-1}$ defined by Eq. (21); if $\left|\Phi_{0}\right\rangle$ belongs to the reduced Hilbert space, it is

$$
\left|\Psi_{0}\right\rangle \equiv \mathcal{U}\left|\Phi_{0}\right\rangle=\sum_{\{s\}} c_{\{s\}}\left|s_{1} s_{2} \cdots s_{N}\right\rangle
$$

with $\{s\} \equiv\left(s_{1}, s_{2}, s_{3} \ldots s_{N}\right)$, and $s_{i} \equiv\left\langle\Psi_{0}\left|S_{i}^{z}\right| \Psi_{0}\right\rangle=+1,0$.

The simplest trial ground state allowing the description of soliton pairs dissociation is that defined by Eq. (22) with $c_{\{s\}}=t_{s_{1} s_{2} s_{3}} t_{s_{2} s_{3} s_{4}} \cdots t_{s_{N-2} s_{N-1} s_{N}}$. The variational parameters are the six amplitudes $t_{+++}, \quad t_{++0}=t_{0++}, \quad t_{+0+}, \quad t_{0+0}, \quad t_{00+}=t_{+00}, \quad t_{000}$, where $\left|t_{s_{i-1} s_{i} s_{i+1}}\right|^{2}$ represents the probability for $\left(S_{i-1}^{z}, S_{i}^{z}, S_{i+1}^{z}\right)$ to be equal $\left(s_{i-1}, s_{i}, s_{i+1}\right)$; a common arbitrary factor may be used for normalizing $\left|\Psi_{0}\right\rangle$. We notice that the chosen form for $c_{\{s\}}$ is such that the probability for $\left|\Psi_{0}\right\rangle$ to contain coupled solitons is finite independently of that relative to the occurrence of isolated solitons, whose presence is unambiguously marked by $t_{+0+} \neq 0$.

Without going into the details of the variational calculations, reported in Ref. 46, we here discuss our final results. Due to the normalization condition, the number of variational parameters is reduced from six to five; moreover, the energy $\left\langle\Psi_{0}\left|\mathcal{U} \widetilde{\mathcal{H}} \mathcal{U}^{-1}\right| \Psi_{0}\right\rangle$ is found to depend just on four precise combinations of the original parameters,

$$
\begin{aligned}
\gamma \equiv\left|t_{++0}\right|^{2}\left|t_{00+}\right|^{2} & \Rightarrow(\cdots++00++\cdots) \\
\pi \equiv\left|t_{++0}\right|^{2}\left|t_{+0+}\right| & \Rightarrow(\cdots++0++\cdots) \\
\chi \equiv\left|t_{00+}\right|^{2}\left|t_{0+0}\right| & \Rightarrow(\cdots 00+00 \cdots) \\
\rho \equiv\left|t_{000}\right| & \Rightarrow(\cdots 000 \cdots)
\end{aligned}
$$

whose square moduli are related to the probabilities that the corresponding strings $(\Rightarrow)$ be contained in $\left|\Psi_{0}\right\rangle$; in particular, $\gamma^{2}$ and $\pi^{2}$ refer to the probabilities for coupled and isolated solitons, respectively, to occur in the ground state. 


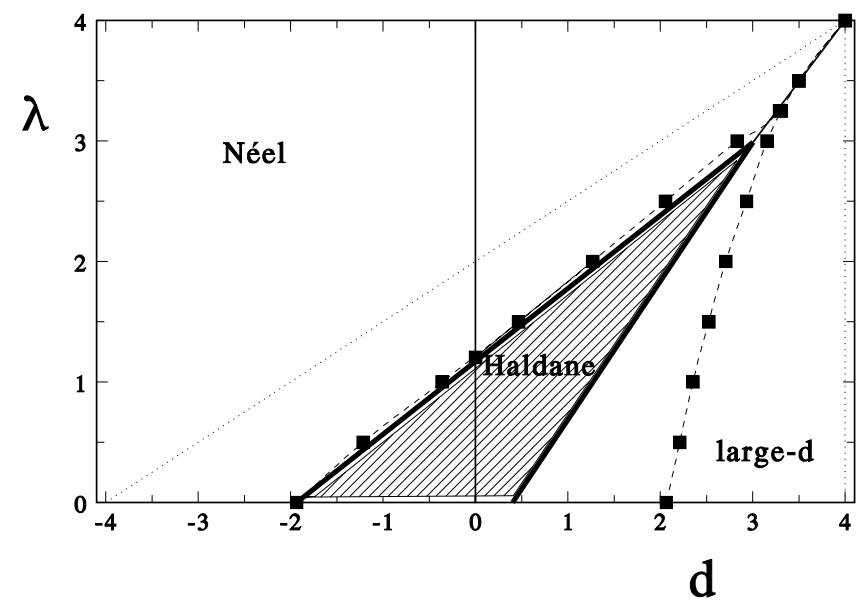

FIG. 6: Phase diagram in the $\lambda>0$ half-plane: our results (squares) are shown together with those of Ref. 43 (dotted lines); the Haldane phase should correspond to the shaded area, according to the best available numerical data ${ }^{33}$ (solid lines).

Both the analytical expression for the energy and the numerical minimization show that it exists a critical value $\lambda_{\mathrm{c}}=\lambda_{\mathrm{c}}(d)>d$ such that, for $\lambda>\lambda_{\mathrm{c}}$ the minimal energy is attained for $\pi=\rho=0$; the condition $\lambda=\lambda_{\mathrm{c}}(d)$ can hence define a curve of phase separation. We therefore single out three different phases, characterized by

(a) $\pi=\rho=0, \quad(b)$ all parameters $\neq 0, \quad(c) \chi=t_{+++}=0$

in the ground state. The corresponding phase diagram is shown in Fig. [6 together with that obtained with a factorized trial ground state ${ }^{43}$, and by numerical simulations 33 .

The $(a)-(b)$ transition is seen to quite precisely describe the Néel-Haldane one, and this leads us to define the condition $(a)$, meaning the occurrence of exclusively coupled solitons, as typical of the Néel phase. As for the $(c)-(b)$ transition, it is to be noticed that the use of the reduced Hilbert space is not fully justified in the $\lambda<d$ region, where we in fact do not expect quantitatively precise results.

As for a comparison between our results and the exact numerical data available, we have considered, along the $d=0$ axis, two specific quantities: the critical anisotropy $\lambda_{c}(d)$, where the Néel phase becomes unstable with respect to the Haldane one, and the ground-state energy $E_{0}(d, \lambda)$ at the isotropic point $\lambda=1$. For the critical anisotropy we find $\lambda_{c}(0)=1.2044(5)$ to be compared with the value obtained by exact diagonalization ${ }^{37}, \lambda_{c}(0) \approx$ 1.19; for the energy we find $E_{0}(0,1)=-1.3663(5)$ to be compared with $E_{0}(0,1)=-1.4014(5)$, again from exact diagonalization technique ${ }^{47}$; the value obtained with the factorized trial ground state is ${ }^{43} E_{0}(0,1)=-4 / 3$.

In Fig. 7 we show the variational parameters as $\lambda$ is varied with $d=0$, i.e. along the $y$ axis of the phasediagram; in fact, rather than the parameters with respect

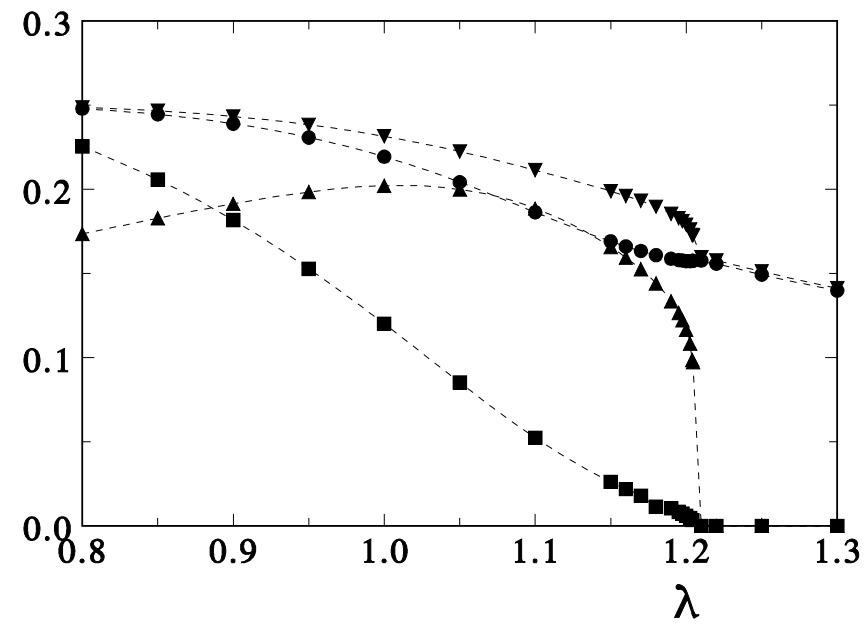

FIG. 7: Parameters $w_{(1)}$ (squares), $w_{(2)}$ (circles), $w_{(3)}$ (upward triangles), and $w_{(2,2)}$ (downward triangles), for $d=0$.

to which we have actually minimized the energy, the following combinations are considered:

$$
\begin{gathered}
w_{(1)}=\pi^{2} / t_{+++}^{2} ; w_{(2)}=\gamma ; \\
w_{(2,2)}=\left(\chi \gamma t_{+++}\right)^{1 / 2} ; w_{(3)}=t_{+++}^{2}(\rho \gamma)^{2 / 3}
\end{gathered}
$$

The above quantities have a straightforward physical meaning, as they are directly related with the probabilities for a soliton to appear along the chain as an isolated excitation $\left(w_{(1)}\right)$, as part of a soliton pair $\left(2 w_{(2)}^{2}\right)$, as part of a string made of three adjacent solitons $\left(3 w_{(3)}^{3}\right)$, and finally as part of a string made of two soliton pairs separated by one site $\left(4 w_{(2,2)}^{4}\right)$. From Fig. 7 it turns evident that the Haldane phase is featured by the occurrence of isolated solitons $\left(w_{(1)} \neq 0\right)$, as well as of strings made of three adjacent solitons $\left(w_{(3)} \neq 0\right)$.

This result confirms that, as elicited by the analysis of the phase-diagram, the Haldane phase is characterized by our condition $(b)$.

Given their essential role, we have also studied the $x$ and $z$ component of the string order parameter, as well as the solitons density $n_{0}=1-\left\langle\left(S^{z}\right)^{2}\right\rangle$. After KT we expect $O_{\text {string }}^{z}(\mathcal{H}) \neq 0$ in both the Néel and the Haldane phase, and $O_{\text {string }}^{x}(\mathcal{H}) \neq 0$ just in the Haldane phase. In fact, analytical expressions for $O^{x}$ and $O^{z}$ may be written ${ }^{46}$ in terms of four of the five variational parameters (25), and show that

- $O_{\text {string }}^{x}(\mathcal{H})=0$ if $\pi=\rho=0$ or $\chi=t_{+++}=0$, i.e. in phase $(a)$ and $(c)$;

- $O_{\text {string }}^{z}(\mathcal{H})>0$ in all phases, asymptotically vanishing as $\rho \rightarrow 1$, i.e. in the far large- $d$ phase.

In more details, we notice that $O_{\text {string }}^{x}=0$ whenever the ground state does not contain strings made of an odd number of adjacent spins; as soon as the shortest string of such type, namely the isolated soliton, appears along the chain, then $O_{\text {string }}^{x}$ gets finite. The unphysical result $O_{\text {string }}^{z}>0$ in the $(c)$ phase, vanishing only as 


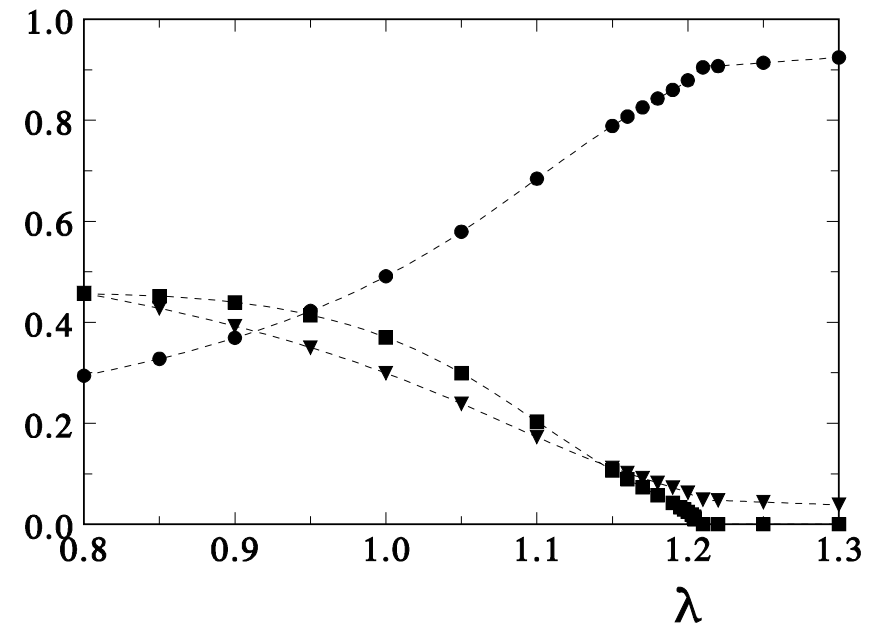

FIG. 8: $\quad$ String order parameters $O_{\text {string }}^{x}$ (squares), $O_{\text {string }}^{z}$ (circles), and solitons density $n_{0}$ (triangles), for $d=0$.

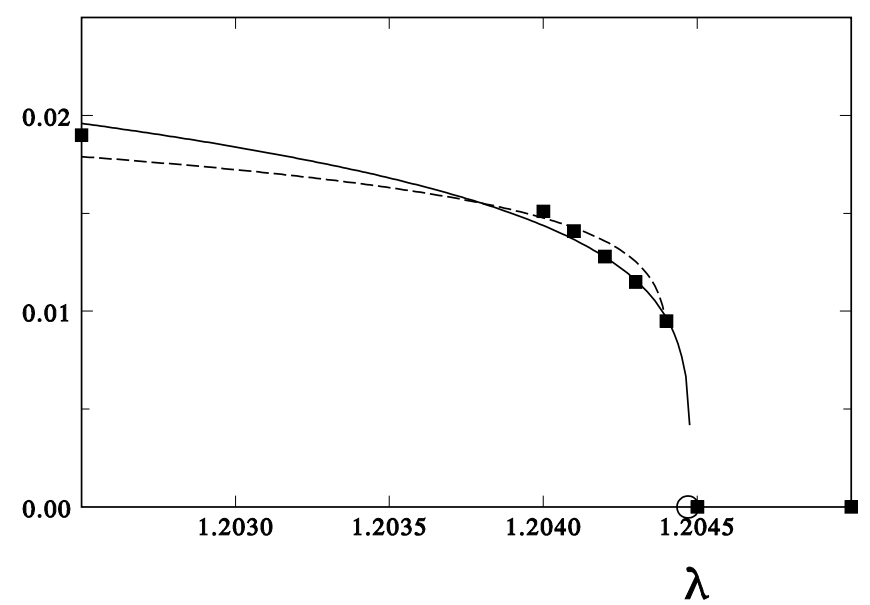

FIG. 9: Critical behavior of $O_{\text {string }}^{x}$ for $d=0$ : squares are results; curves are obtained by best-fit procedure from $O_{\text {string }}^{x} \sim\left(\lambda-\lambda_{c}\right)^{\beta}$ with $\beta$ fixed to 0.125 (dashed curve), and as fitting parameter, resulting in $\beta=0.217$ (solid curve). Both procedures give $\lambda_{\mathrm{c}}=1.2044(5)$, marked by a circle in figure.

$d \rightarrow \infty$ rather than everywhere in the large- $d$ phase, is due to our assuming the ground state to belong to the reduced Hilbert space, which is actually licit just in the $\lambda>d$ region.

In Fig. 8 we show $O_{\text {string }}^{x}, O_{\text {string }}^{z}$, and $n_{0}$ as $\lambda$ varies with $d=0$ : We underline that $O_{\text {string }}^{x}$ gets finite continuously but with discontinuous derivative at the transition (reflecting the behavior of $w_{(1)}$ and $w_{(3)}$ shown in Fig. (7), so that the Néel-Haldane quantum phase transition is recognized as a second order one. In Fig. 9 we zoom the order parameter $O_{\text {string }}^{x}$ around the critical point: its behavior is seen to be described by a power law $O_{\text {string }}^{x} \sim\left(\lambda_{c}-\lambda\right)^{\beta}$, as expected for a continuous phase transition; our estimated value for the critical exponent is $\beta=0.217(5)$ to be compared with $\beta=0.125$, corresponding to the Ising model in a transverse field, to whose universality class the Haldane transition is suggested to belong to ${ }^{42}$. At the isotropic point $(d=0, \lambda=1)$ we find $O_{\text {string }}^{x}=0.3700(5)$ in full agreement with the value obtained by exact diagonalization ${ }^{48}$.

The overall good agreement between our results and the numerical available data, allows us to conclude that the Néel-Haldane transition is a second-order one, and that the string order parameter $O_{\text {string, }}^{x}$, revealing hidden order along the $x$ direction, is the appropriate order parameter for the Haldane phase. The disordered ground state featuring the Haldane phase is seen to originate by soliton pairs dissociation, according to this path: Solitons occur just in pairs in the antiferromagnetically ordered Néel phase; at the Néel-Haldane transition soliton pairs dissociate and the byproducts rearrange in strings made of an odd number of solitons. These strings are ultimately responsible for the disorder of the ground state.

\section{TWO-DIMENSIONAL ISOTROPIC HEISENBERG MODEL}

The 2D isotropic QHAF on the square lattice is one of the magnetic models most intensively investigated in the last two decades. This is due both to its theoretically challenging properties and to its being considered the best candidate for modeling the magnetic behavior of the parent compounds of some high- $T_{\mathrm{c}}$ superconductors 2.3 .

From a theoretical point of view the fully isotropic Heisenberg model in $d$ dimensions, thanks to the simple structure of its Hamiltonian (whose high symmetry is responsible for most of its peculiar features), may well be considered a cornerstone of the modern theory of critical phenomena, with its relevance extending well beyond the only magnetic systems. The $d=2$ case earned additional interest, representing the boundary dimension separating systems with and without long-range order at finite temperature ${ }^{4}$. The antiferromagnetic coupling adds further appeal, as the classical-like Néel state is made unstable by quantum fluctuations and the ground state of the system is not exactly known. It can be rigorously proven 49 to be ordered for $S \geq 1$; for $S=1 / 2$ there is no rigorous proof, although evidences for an ordered ground state can be drawn from many different studies (for a review, see, for instance, Ref. 50 ).

On the experimental side the attention on the properties of $2 \mathrm{D}$ QHAF was mainly triggered by the fact that among the best experimental realizations of this model we find several parent compounds of high- $T_{\mathrm{c}}$ superconductors, as, e.g., $\mathrm{La}_{2} \mathrm{CuO}_{4}$ or $\mathrm{Sr}_{2} \mathrm{CuO}_{2} \mathrm{Cl}_{2}$ 51.52.53, both having spin $S=1 / 2$. In such materials, as well as in other magnetic compounds with a layered crystal structure as $\mathrm{La}_{2} \mathrm{NiO}_{4}{ }^{54}$ and $\mathrm{K}_{2} \mathrm{NiF}_{4}{ }^{52.53}(S=1), \mathrm{Rb}_{2} \mathrm{MnF}_{4}{ }^{55}$ and $\mathrm{KFeF}_{4}{ }^{56}(S=5 / 2)$ or copper formate tetradeuterate (CFTD, $S=1 / 2)^{57}$ the magnetic ions form parallel planes and interact strongly only if belonging to the same plane. The interplane interaction in these compounds is several orders of magnitude smaller than the intra- 
plane one, thus offering a large temperature region where their magnetic behavior is indeed $2 \mathrm{D}$ down to those low temperatures where the weak interplane interaction becomes relevant, driving the system towards a $3 \mathrm{D}$ ordered phase: an antiferromagnetic Heisenberg interaction and the small spin value make these compounds behave as 2D QHAFs. Even the onset of 3D magnetic long-range order is however strongly affected by the $2 \mathrm{D}$ properties of the system: indeed, the observed 3D magnetic transition temperature is comparable with the intraplane interaction energy, i.e., several order of magnitude larger than that one can expect only on the basis of the value of the interplane coupling. Such apparently odd behavior can be easily understood by observing that the establishing of in-plane correlations on a characteristic distance $\xi$ effectively enhances the interplane coupling by a factor $(\xi / a)^{2}, a$ being the lattice constant. The latter consideration is one of the reasons explaining why most of the attention, both from the experimental and theoretical point of view, was devoted to the low-temperature behavior of the correlation length $\xi$ of the 2D QHAF (in the following $\xi$ will be always given in units of the lattice constant $a$ ).

The 2D QHAF is described by the Hamiltonian

$$
\mathcal{H}=\frac{J}{2} \sum_{i, d} \boldsymbol{S}_{i} \cdot \boldsymbol{S}_{\boldsymbol{i}+\boldsymbol{d}}
$$

where $J$ is positive and the quantum spin operators $\boldsymbol{S}_{\boldsymbol{i}}$ satisfy $\left|\boldsymbol{S}_{\boldsymbol{i}}\right|^{2}=S(S+1)$. The index $\boldsymbol{i} \equiv\left(i_{1}, i_{2}\right)$ runs over the sites of a square lattice, and $\boldsymbol{d}$ represents the displacements of the 4 nearest-neighbors of each site, $( \pm 1,0)$ and $(0, \pm 1)$.

In addition to the first approximations usually employed to investigate the low temperature properties of magnetic systems as, e.g., mean-field and (modified) spin-wave theory, the critical behavior of the 2D QHAF was commonly interpreted on the basis of the results obtained by field theory starting from the so-called $2 \mathrm{D}$ quantum nonlinear $\sigma$ model $(\mathrm{QNL} \sigma \mathrm{M})^{58}$, whose action is given by

$$
S=\frac{1}{2 g} \int d \boldsymbol{x} \int_{0}^{u} d \tau\left(|\nabla \boldsymbol{n}|^{2}+\left|\partial_{\tau} \boldsymbol{n}\right|^{2}\right) ; \quad|\boldsymbol{n}|^{2}=1 .
$$

In the last Equation $\boldsymbol{n}(\boldsymbol{x})$ is a unitary 3D vector field, $g=c \Lambda / \rho$ and $u=c \Lambda / T$ are the coupling and the imaginary-time cut-off respectively, and the two parameters $\rho$ and $c$ are usually referred to as spin stiffness and spin-wave velocity. Despite their names, the two parameters $\rho$ and $c$ are however just phenomenological fitting constants which can be rigorously related to the proper parameter $J$ and $S$ of the original magnetic Hamiltonian (26) only in the large- $S$ limit 1.59 . The source of non-linearity in the model Eq. (27), which is seemingly quadratic in the field variables, is the constraint imposed onto the length of the field $\boldsymbol{n}$.

The relation between the 2D QHAF and the QNL $\sigma \mathrm{M}$ was first exploited to interpret the experimental data on cuprous oxides by Chakravarty, Halperin, and Nelson ${ }^{58}$ (CHN) who used symmetry arguments to show that the long-wavelength physics of the QHAF is the same of that of the QNL $\sigma \mathrm{M}$; in other words the physical observables of the two models show the same functional dependence upon $T$, if the long-wavelength excitations are assumed to be the only relevant ones, as one expects to be at low temperature.

The analysis carried out by CHN on the QNL $\sigma \mathrm{M}$ lead to single out three different regimes, called quantum disordered, quantum critical (QCR) and renormalized classical (RCR), the most striking difference amongst them being the temperature dependence of the spin correlations. If $g$ is such as to guarantee LRO at $T=0$, the $\mathrm{QNL} \sigma \mathrm{M}$ is in the RCR at very low-temperature and the correlation length $\xi$ behaves as ${ }^{60}$ :

$$
\xi_{31}=\frac{e}{8}\left(\frac{c}{2 \pi \rho}\right) \exp \left(\frac{2 \pi \rho}{T}\right)\left[1-\frac{T}{4 \pi \rho}\right] .
$$

CHN found also that by raising the temperature any 2D QNL $\sigma \mathrm{M}$ with an ordered ground state crosses over from the RCR to the QCR, characterized by a correlation length $\xi \propto \alpha(T)=c / T$.

The first direct comparison between experimental data on spin 1/2 compounds and the prediction of the QNL $\sigma \mathrm{M}$ field theory in the RCR gave surprisingly good agreement and caused an intense activity, both theoretical and experimental, in the subsequent years. However, with the accumulation of new experimental data on higher spin compounds it clearly emerged that the experimentally observed behavior of $\xi(T)$ for larger spin could not be reproduced neither by the original simplified (2-loop) form of Eq. (28) given by CHN (which does not contain the term in square brackets), nor by the three-loops result (28) derived by Hasenfratz and Niedermayer 60 (HN); moreover no trace of QCR behavior was found in pure compounds. The discrepancies observed could be due to the fact that the real compounds do not behave like 2D QHAF or to an actual inadequacy of the theory. In particular the CHN-HN scheme introduces two possible reasons for such inadequacy to occur: the physics of the 2D QHAF is not properly described by that of the $2 \mathrm{D} \mathrm{QNL} \sigma \mathrm{M}$ and/or the two(three)-loop renormalizationgroup expressions derived by CHN-HN do hold at temperatures lower than those experimentally accessible. After an almost ten years long debate, the latter possibility has finally emerged as the correct one, being strongly supported not only by our own work, but also by other independent theoretical approaches $\frac{61}{1}$, joined with the analysis of the experimental and the most recent quantum Monte Carlo (QMC) data for the 2D QHAF.

The theoretical approach we employed to investigate the 2D QHAF is the effective Hamiltonian method ${ }^{62.63 .64}$, developed within the framework of the pure-quantum self-consistent harmonic approximation (PQSCHA) we introduced at the beginning of the 90's ${ }^{65.66}$. The PQSCHA starts from the Hamiltonian path-integral formulation of statistical mechanics which 
allows one to separate in a natural way classical and quantum fluctuations: only the latter are then treated in a self-consistent harmonic approximation, finally getting an effective classical Hamiltonian, whose properties can thereafter be investigated by all the techniques available for classical systems. The idea of separating classical and quantum fluctuations turned out to be fruitful not only in view of the implementation of the PQSCHA, but also in the final understanding of the connection between semiclassical approaches and quantum field theories 67 , which could be possible also thanks to the paper by Hasenfratz $^{68}$ about corrections to the field-theoretical results due to cutoff effects.

The PQSCHA naturally applies to bosonic systems, whose Hamiltonian is written in terms of conjugate operators $\hat{q} \equiv\left(\hat{q}_{1}, \ldots \hat{q}_{N}\right), \underline{\hat{p}} \equiv\left(\hat{p}_{1}, \ldots \hat{p}_{N}\right)$ such that $\left[\hat{q}_{m}, \hat{p}_{n}\right]=i \bar{\delta}_{m n}$; the method, however, does not require $\mathcal{H}(\underline{\hat{p}}, \underline{\hat{q}})$ to be standard, i.e., with separate quadratic kinetic $\underline{\hat{p}}$-dependent and potential $\hat{q}$-dependent terms, and its application may be extended also to magnetic systems, according to the following scheme ${ }^{65}$ : The spin Hamiltonian $\mathcal{H}(\underline{\boldsymbol{S}})$ is mapped to $\mathcal{H}(\underline{\hat{p}}, \underline{\hat{q}})$ by a suitable spin-boson transformation; once the corresponding Weyl symbol $\mathcal{H}(\underline{p}, \underline{q})$, with $\underline{p} \equiv\left(p_{1}, \ldots p_{N}\right)$ and $\underline{q} \equiv\left(q_{1}, \ldots q_{N}\right)$ classical phase-space variables, has been determined, the PQSCHA renormalizations may be evaluated and the effective classical Hamiltonian $\mathcal{H}_{\mathrm{eff}}(\underline{p}, \underline{q})$ and effective classical function $\mathcal{O}_{\text {eff }}(\underline{p}, \underline{q})$ corresponding to the observable $\mathcal{O}$ of interest follow. Finally the effective functions $\mathcal{H}_{\text {eff }}(\underline{s})$ and $\mathcal{O}_{\text {eff }}(\underline{s})$, both depending on classical spin variables $\boldsymbol{s}$ with $|\boldsymbol{s}|=1$ and containing temperature- and spin-dependent quantum renormalized parameters, are reconstructed by the inverse of the classical analogue of the spin-boson transformation used at the beginning.

In order to successfully carry out such renormalization scheme, the Weyl symbol of the bosonic Hamiltonian must be a well-behaved function in the whole phase space. Spin-boson transformations, on the other hand, can introduce singularities as a consequence of the topological impossibility of a global mapping of a spherical phase space into a flat one. The choice of the transformation must then be such that the singularities occur for configurations which are not thermodynamically relevant, and whose contribution may be hence approximated. Most of the methods for studying magnetic systems do in fact share this problem with the PQSCHA; what makes the difference is that by using the PQSCHA one separates the classical from the pure-quantum contribution to the thermal fluctuations, and the approximation only regards the latter, being the former exactly taken into account when the effective Hamiltonian is recast in the form of a classical spin Hamiltonian.

The spin-boson transformation which constitutes the first step of the magnetic PQSCHA is chosen according to the symmetry properties of the original Hamiltonian and of its ground state. In the case of the 2D QHAF both Dyson-Maleev and Holstein-Primakoff transforma- tion can be employed finally obtaining 63 :

$$
\begin{aligned}
\frac{\mathcal{H}_{\mathrm{eff}}}{J \widetilde{S}^{2}} & =\frac{\theta^{4}}{2} \sum_{\boldsymbol{i}, \boldsymbol{d}} \boldsymbol{s}_{\boldsymbol{i}} \cdot \boldsymbol{s}_{\boldsymbol{i}+\boldsymbol{d}}+N \mathcal{G}(t) \\
\mathcal{G}(t) & =\frac{t}{N} \sum_{\boldsymbol{k}} \ln \frac{\sinh f_{k}}{\theta^{2} f_{k}}-2 \kappa^{2} \mathcal{D}
\end{aligned}
$$

with the temperature and spin dependent parameters

$$
\begin{aligned}
\theta^{2} & =1-\frac{\mathcal{D}}{2} \\
\mathcal{D} & =\frac{1}{\widetilde{S} N} \sum_{\boldsymbol{k}}\left(1-\gamma_{\boldsymbol{k}}^{2}\right)^{\frac{1}{2}} \mathcal{L}_{\boldsymbol{k}} \\
f_{\boldsymbol{k}} & =\frac{\omega_{\boldsymbol{k}}}{2 \widetilde{S} t}, \quad \mathcal{L}_{\boldsymbol{k}}=\operatorname{coth} f_{\boldsymbol{k}}-\frac{1}{f_{\boldsymbol{k}}} .
\end{aligned}
$$

In the previous Equations $\gamma_{\boldsymbol{k}}=\left(\cos k_{1}+\cos k_{2}\right) / 2, N$ is the number of sites of the lattice and $\boldsymbol{k} \equiv\left(k_{1}, k_{2}\right)$ is the wave-vector in the first Brillouin zone; $\widetilde{S} \equiv S+1 / 2$ is the effective classical spin length, which naturally follows from the renormalization scheme, and $t \equiv T / J \widetilde{S}^{2}$ is the reduced temperature defined in terms of the energy scale $J \widetilde{S}^{2}$. The renormalization scheme is closed by the self-consistent solution of the two coupled equations $\omega_{\boldsymbol{k}}=4 \kappa^{2}\left(1-\gamma_{\boldsymbol{k}}^{2}\right)^{1 / 2}$ and $\kappa^{2}=\theta^{2}-t /\left(2 \kappa^{2}\right)$. The purequantum renormalization coefficient $\mathcal{D}=\mathcal{D}(S, t)$ takes the main contribution from the high-frequency part (shortwavelength) of the spin-wave spectrum, because of the appearance of the Langevin function $\mathcal{L}_{\boldsymbol{k}} . \mathcal{D}$ measures the strength of the pure-quantum fluctuations, whose contribution to the thermodynamics of the system is the only approximated one in the PQSCHA scheme. The theory is hence quantitatively meaningful as far as $\mathcal{D}$ is small enough to justify the self-consistent harmonic treatment of the pure-quantum effects. In particular, the simple criterion $\mathcal{D}<0.5$ is a reasonable one to assess the validity of the final results.

The most relevant information we get from Eq. (29) is that the symmetry of the Hamiltonian is left unchanged so that from a macroscopic point of view the quantum system essentially behaves, at an actual temperature $t$, as its classical counterpart does at an effective temperature $t_{\text {eff }}=t / \theta^{4}(S, t)$. This allows us to deduce the behavior of many observables (but not all!, see Refs. 626364 for details) directly from the behavior of the corresponding classical quantities. This is the case of the correlation length, which turns out to be given simply by:

$$
\xi(t)=\xi_{\mathrm{cl}}\left(t_{\mathrm{eff}}\right)
$$

so that once $\theta^{4}(S, t)$ has been evaluated, the only additional information we need is the classical $\xi_{\mathrm{cl}}(t)$, which is available from classical Monte Carlo simulation and analytical asymptotic expressions ${ }^{69}$ as $t \rightarrow 0$.

Sample results obtained by PQSCHA are shown in the figures. In Fig. 10 the correlation length for $S=1 / 2$ and $S=1$ is compared with experimental data; a similar comparison, including $\mathrm{MC}$ data for $S=1 / 2$ and experimental data on $S=5 / 2$ compounds $\mathrm{KFeF}_{4}$ and $\mathrm{Rb}_{2} \mathrm{MnF}_{4}$ is 


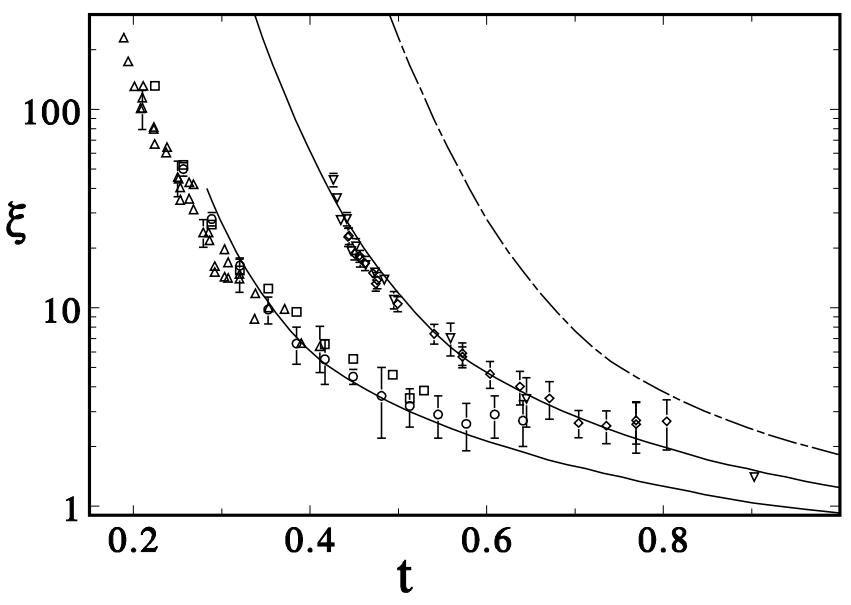

FIG. 10: Correlation length $\xi$ vs $t$, for $S=1 / 2$ (leftmost) and $S=1$. The symbols are experimental data; for $S=1 / 2:{ }^{63} \mathrm{Cu}$ NQR data ${ }^{70}$ (circles) and neutron scattering data for $\mathrm{La}_{2} \mathrm{CuO}_{4}$ (squares ${ }^{51}$ ) and for $\mathrm{Sr}_{2} \mathrm{CuO}_{2} \mathrm{Cl}_{2}$ (uptriangles ${ }^{52,53}$ ); for $S=1$ : neutron scattering data for $\mathrm{La}_{2} \mathrm{NiO}_{4}$ (down-triangles ${ }^{54}$ ) and for $\mathrm{K}_{2} \mathrm{NiF}_{4}$ (diamonds ${ }^{52.53}$ ). The classical result (dash-dotted line) is also reported.

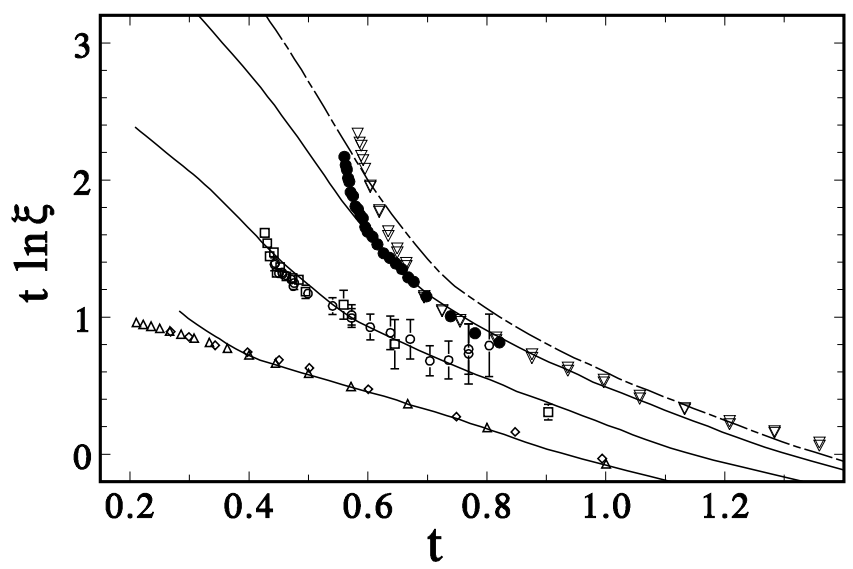

FIG. 11: The function $y(t)=t \ln \xi$ vs $t$, for (from the rightmost curve) $S=\infty, 5 / 2,1$ and $1 / 2$; the up-triangles ${ }^{71}$ and the diamonds ${ }^{72}$ are quantum MC data for $S=1 / 2$; also reported are neutron scattering data for $\mathrm{La}_{2} \mathrm{NiO}_{4}$ (open circles ${ }^{54}$ ), for $\mathrm{K}_{2} \mathrm{NiF}_{4}$ (squares ${ }^{52,53}$ ), $\mathrm{KFeF}_{4}$ (filled circles ${ }^{56}$ ) and $\mathrm{Rb}_{2} \mathrm{MnF}_{4}$ (down-triangles ${ }^{55}$ ). The abrupt rising of the experimental data for the $S=5 / 2$ compounds at $t \simeq 0.65$ is due to the effect of the small, but finite, anisotropies and will be discussed in more details in Section $\mathrm{V}$

made in Fig. 11] but along the vertical axis the quantity $t \ln \xi$ is reported in order to better appreciate the deviation from the predicted RCR behavior, that would correspond to a straight line at low $t$. From the last picture one can easily see that both PQSCHA curves and experimental data for $S \geq 1$ (including the exact $S=\infty$ classical result) display a change of slope at intermediate temperature, followed by a curvature inversion at lower $t$. On the other hand, by looking at the $S=1 / 2$ case it becomes clear why the QNL $\sigma \mathrm{M}$ approach gave such a

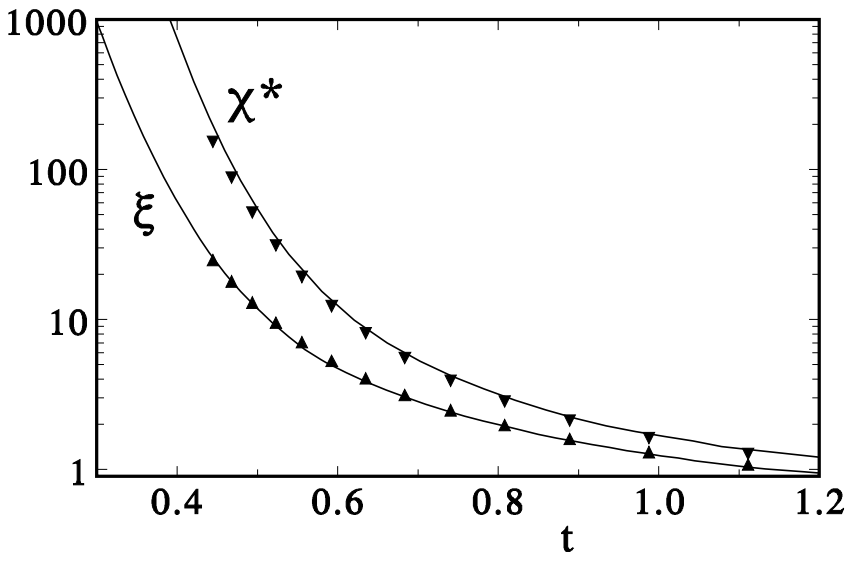

FIG. 12: Correlation length $\xi$ and staggered susceptibility $\chi^{*} \equiv \chi / \widetilde{S}^{2}$ vs $t$ for $S=1$. Symbols are QMC data from Ref. 74 .

good agreement when firstly used to fit the experimental data. The change in both the slope and the curvature of $t \ln \xi$ is less pronounced and possibly occurs at lower temperatures, the lower the spin: in the $S=1 / 2$ case, it is difficult to say whether these features are still present or not, but, if yes, they occur in a temperature region where the extremely high value of $\xi\left(\approx 10^{4}\right)$ makes both the experimental and the simulation data more difficult to be obtained.

After having realized that the field theoretical prediction by CHN could not be applied to the $S \geq 12 \mathrm{D} \mathrm{QHAF}$ in the temperature range probed by the experiments, the following questions were waiting for a satisfactory answer: $(i)$ the real range of applicability of the asymptotic three-loop expression (28) at different $S$, and (ii) the possible extension of the PQSCHA results to lower temperature, both in view of (iii) a comprehensive description of the behavior of the correlation length of the $2 \mathrm{D}$ QHAF in the entire range of temperature and spin values.

A substantial contribution to settle this conundrum came only from QMC simulations for higher spin values able to probe the very large correlation length region ${ }^{73}$ : indeed, high precision Monte Carlo data for $S=1$ and moderate correlation length could still be very well interpreted by PQSCHA and did not display the RCR asymptotic behavior, as shown ${ }^{64}$ in Fig. 12 where we compared our curves for $\xi$ and staggered susceptibility $\chi^{*}$ with QMC data obtained by Harada et al ${ }^{74}$.

QMC results for $\xi$ by Beard and coworkers ${ }^{73}$ showed unambiguously that the three-loop Eq. (28) holds only for temperatures low enough to ensure an extremely large correlation length, e.g., $\xi \gtrsim 10^{5}$ for $S=1, \xi \gtrsim 10^{12}$ for $S=3 / 2$, and generally cosmological correlation lengths for $S>3 / 2$, thus definitely excluding any possibility of employing QNL $\sigma \mathrm{M}$ results to interpret available experimental data.

In Ref. 68 Hasenfratz showed why cutoff effects, which are so devious for $S=1 / 2$, significantly modify the corre- 


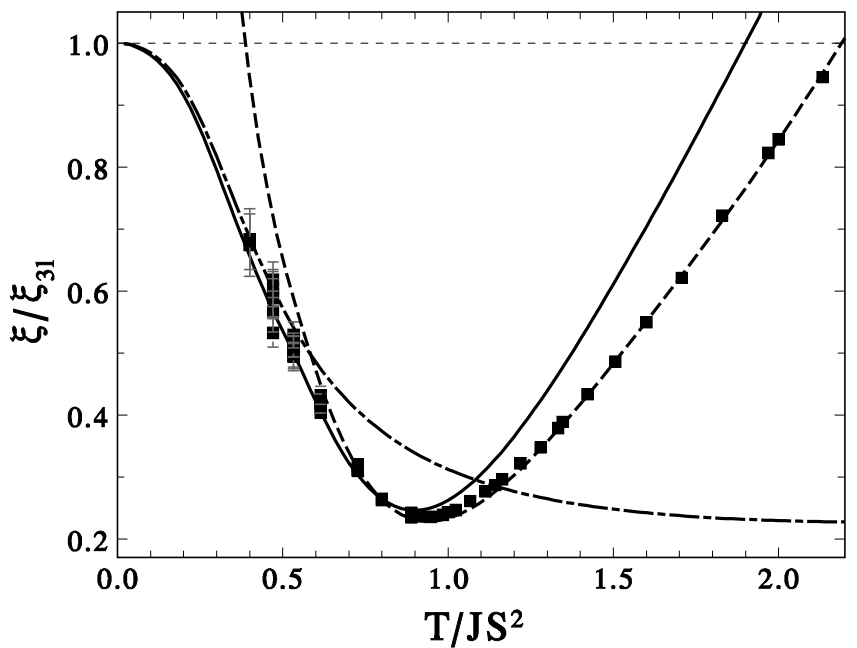

FIG. 13: Ratio $\xi / \xi_{31}$ vs $T / J S^{2}$ for $S=5 / 2$. Solid line: $\xi_{\mathrm{cl}}\left(t_{\mathrm{eff}}^{\mathrm{H}}\right)$; dash-dotted line: $\xi_{\mathrm{H}} \simeq \xi_{31}^{\mathrm{cl}}\left(t_{\mathrm{eff}}^{\mathrm{H}}\right)$; dashed line: standard PQSCHA result; symbols are QMC data ${ }^{67}$.

lation length for $S \geq 1$. Reaching such goal was possible only going back to a direct mapping between the QHAF and the QNL $\sigma \mathrm{M}$, and the resulting cutoff-corrected fieldtheoretical outcome is 68

$$
\xi_{\mathrm{H}}(T, S)=\xi_{31}(T, S) e^{-C(T, S)},
$$

where $C(T, S)$, defined in Eq. (14) of Ref. 68, is an integral of familiar spin-wave quantities over the first Brillouin zone.

With this correction, which is the leading order in the spin-wave expansion for the cutoff correction, it is possible to obtain numerically accurate agreement with QMC data down to $\xi \gtrsim 10^{3}$ for all $S$.

In our most recent paper $\frac{67}{}$ about $2 \mathrm{D}$ QHAF we showed that by employing the explicit expression for $C(T, S)$, and by substituting in Eq. (28) the spin stiffness $\rho$ and the spin-wave velocity $c$ given by the mapping of QHAF onto the QNL $\sigma \mathrm{M}$, the leading terms of the result (35) not only can be cast into the form $\xi_{\mathrm{H}}(T, S)=\xi_{31}^{\mathrm{cl}}\left(t_{\mathrm{eff}}^{\mathrm{H}}\right)$, in strict analogy with the PQSCHA expression (34), but the effective temperature $t_{\mathrm{eff}}^{\mathrm{H}}$ is defined through a renormalization constant which is again a function of the pure-quantum fluctuations only! Such remarkable and unexpected feature of the cutoff-corrected field-theory prediction, suggested us to substitute the perturbative expression $\xi_{31}^{\mathrm{cl}}$ with the exactly known classical $\xi_{\mathrm{cl}}$ thus getting the results presented in Fig. [13]

It is thus made clear that the main features of the quantum correlation length at intermediate temperatures are due to essentially classical non-linear effects, which cannot be taken into account by perturbative approaches. Moreover, the effective exchange constant which defines the effective temperature $t_{\mathrm{eff}}^{\mathrm{H}}$ is seen to depend on the same pure-quantum renormalization coefficients defined by the PQSCHA, according to an expression which is very similar (equal) to that found by the latter approach in its standard (low- $T$ ) version: the behavior of $\xi$ in the full temperature and spin-value ranges can thus be quantitatively described by Eq. (34) without any adjustable fitting parameter.

The results obtained by the PQSCHA about the correlation length and other static quantities can also represent the needed information to be inserted within other frameworks, like mode-coupling theory, to interpret experiments probing dynamic quantities, like nuclear magnetic resonance (NMR): an example of the successful combination of PQSCHA and mode-coupling theory is given in Ref. 57 .

\section{TWO-DIMENSIONAL ANISOTROPIC HEISENBERG MODEL}

While the theoretical debate mentioned in the previous Section has been mainly dedicated to the isotropic 2D QHAF, real compounds are not actually well described by the isotropic model when the temperature is low: indeed, the Mermin-Wagner theorem ${ }^{4}$ states that a finitetemperature transition cannot occur in the $2 \mathrm{D}$ isotropic QHAF, while the experimental evidence of a transition suggests that 3D correlations and anisotropy effects, as well as a combination of both, must be considered. Easyaxis (EA) or easy-plane (EP) anisotropies turn out to be fundamental in the analysis of the critical behavior.

\section{A. 2D antiferromagnet with easy-axis anisotropy}

Several works (see Ref. 75 for a review) have shown that many additional interaction mechanisms may be taken into account by inserting proper anisotropy terms in the magnetic Hamiltonian; in particular, the transition observed in $\mathrm{K}_{2} \mathrm{NiF}_{4}{ }^{76}(S=1), \mathrm{Rb}_{2} \mathrm{FeF}_{4}{ }^{76}(S=2)$, $\mathrm{K}_{2} \mathrm{MnF}_{4}{ }^{77}, \mathrm{Rb}_{2} \mathrm{MnF}_{4}{ }^{76}(S=5 / 2)$, and others, is seen to be possibly due to an easy-axis anisotropy. Such anisotropy has been often described in the literature through an external staggered magnetic field in order to allow for a qualitative description of the experimental data. However, this choice lacks the fundamental property of describing a genuine phase transition, as the field explicitly breaks the symmetry and makes the model ordered at all temperatures. To preserve the symmetry under inversion along the easy-axis, it is actually appropriate to insert an exchange anisotropy term in the spin Hamiltonian. Spontaneous symmetry breaking manifests then itself as a phase transition between ordered and disordered states. The EA-QHAF Hamiltonian reads then

$$
\mathcal{H}=\frac{J}{2} \sum_{i, \boldsymbol{d}}\left[\mu\left(S_{\boldsymbol{i}}^{x} S_{\boldsymbol{i}+\boldsymbol{d}}^{x}+S_{\boldsymbol{i}}^{y} S_{\boldsymbol{i}+\boldsymbol{d}}^{y}\right)+S_{\boldsymbol{i}}^{z} S_{\boldsymbol{i}+\boldsymbol{d}}^{z}\right]
$$

where $\boldsymbol{i}=\left(i_{1}, i_{2}\right)$ runs over the sites of a square lattice, $\boldsymbol{d}$ connects each site to its four nearest neighbors, $J>0$ is 
the exchange integral and $\mu$ is the easy-axis anisotropy parameter $(0 \leq \mu<1)$. Again, $J \widetilde{S}^{2} \equiv J(S+1 / 2)^{2}$ sets the overall energy scale and $t=T / J \widetilde{S}^{2}$ is the reduced temperature. When $\mu=1$ the model reduces to the isotropic QHAF. Note that a canonical transformation reversing the $x$ and $y$ spin components one one sublattice is equivalent to setting $\mu \rightarrow-\mu$, so that the physical properties of the model are even functions of $\mu$. The $\mu=0$ case is called Ising limit, not to be confused with the genuine Ising mode178, reproduced by Eq. (36) with $\mu=0$ and $S=1 / 2$. Despite being a very particular case of Eq. (36), the $2 \mathrm{D}$ Ising model on the square lattice is a fundamental point of reference for the study of the thermodynamic properties of the EA-QHAF. A renormalization-group analysis 79 of the classical model predicted the occurrence of an Ising-like transition at a finite temperature $t_{\mathrm{c}}^{\mathrm{cl}}(\mu)$ of the order of unity for any value of $\mu$, no matter how near to the isotropic value $\mu=1$; this analysis received the support of several Monte Carlo simulations ${ }^{80.81 .82 .83}$.

As for the quantum case, up to a few years ago no information was available about the value of the critical temperature $t_{\mathrm{c}}(\mu, S)$ as a function of anisotropy and spin, save the fact that $t_{\mathrm{c}}(0,1 / 2)=0.567 \mathrm{~J}$ (Onsager solution ${ }^{78}$ ) and $t_{\mathrm{c}}(1, S)=0$ (isotropic limit). As a consequence it was also uncertain whether or not the small anisotropy $\left(1-\mu \simeq 10^{-2}\right)$ observed in real compounds could be responsible of transitions occurring at critical temperatures of the order of $J$, also accounting for the fact that quantum fluctuations are expected to lower the critical temperature with respect to the classical case.

Over the last few years our group developed a quantitative analysis of several thermodynamic properties of the model, by means of the effective Hamiltonian method ${ }^{65.66}$ for spin $S \geq 1$ and by means of quantum Monte Carlo simulations ${ }^{84}$ in the case $S=1 / 2$.

The effective Hamiltonian ${ }^{85.86 .87}$ for the EA-QHAF is expressed for classical spins as

$$
\frac{\mathcal{H}_{\mathrm{eff}}}{J \widetilde{S}^{2}}=\frac{j_{\mathrm{eff}}}{2} \sum_{\boldsymbol{i}, \boldsymbol{d}}\left[\mu_{\mathrm{eff}}\left(s_{\boldsymbol{i}}^{x} s_{\boldsymbol{i}+\boldsymbol{d}}^{x}+s_{\boldsymbol{i}}^{y} s_{\boldsymbol{i}+\boldsymbol{d}}^{y}\right)+s_{\boldsymbol{i}}^{z} s_{\boldsymbol{i}+\boldsymbol{d}}^{z}\right]
$$

and shows a weaker renormalized exchange $j_{\text {eff }}(t, \mu)<1$ and easy-axis anisotropy $\mu_{\mathrm{eff}}(t, \mu)>\mu$, besides an additional free-energy term that is not reported. By means of $\mathcal{H}_{\mathrm{eff}}$ a series of thermodynamic quantities were studied $^{85,88}$ : internal energy, specific heat, staggered magnetization, staggered correlation function, staggered correlation length, staggered susceptibility. This required extensive classical Monte Carlo simulations, as varying the temperature gives an effective system with different effective anisotropy $\mu_{\text {eff }}(t, \mu)$. The quantum phase diagram reported in Fig. 14 could be built up ${ }^{87.89}$ in a simpler way starting from the knowledge of the classical one and using a relation that follows from the form of Eq. (37),

$$
t_{\mathrm{c}}(\mu, S)=j_{\mathrm{eff}}(t, \mu, S) t_{\mathrm{c}}^{\mathrm{cl}}\left(\mu_{\mathrm{eff}}(t, \mu, S)\right) .
$$

In the region of very weak anisotropy, which is the most

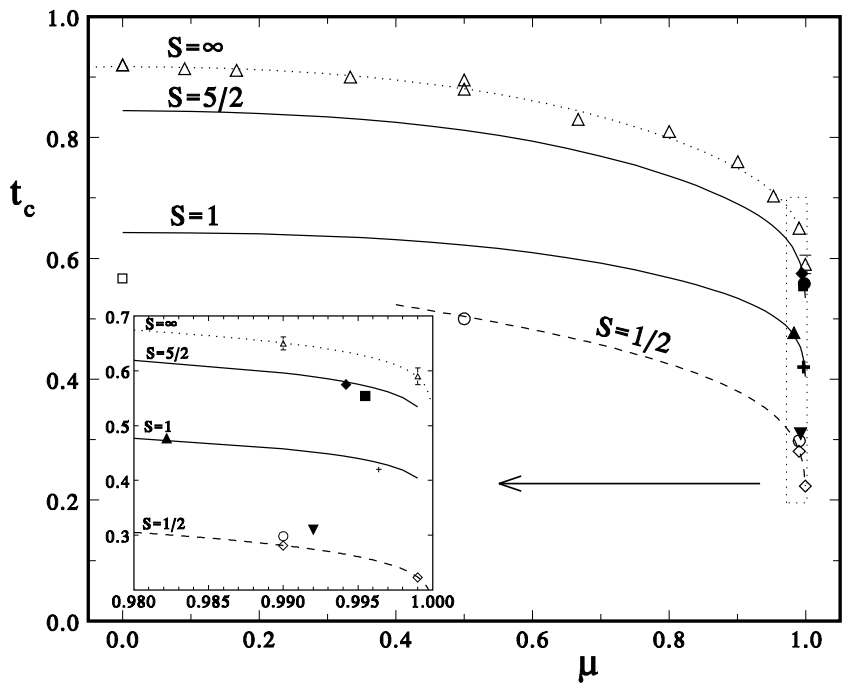

FIG. 14: Critical temperature vs anisotropy $\mu$ for the 2D easy-axis antiferromagnet. Dotted line: the fit of $t_{\mathrm{c}}^{\mathrm{cl}}(\mu)$ build up from classical MC data ${ }^{82.83 .87}$ (open triangles). Solid lines: PQSCHA result for $S=1$ and $5 / 2$. Quantum MC data (open $\operatorname{circles}^{90}$ and diamonds ${ }^{84}$ ) for $S=1 / 2$, asymptotically described by $t_{\mathrm{c}}(\mu)=2.49 / \ln [70 /(1-\mu)]$ (dashed line), and exact result for the Ising model, $\mu=0$ (open square). Experimental data for the compounds $\mathrm{YBa}_{2} \mathrm{Cu}_{3} \mathrm{O}_{6} .1$ (down triangle $\left.{ }^{91}\right), \mathrm{K}_{2} \mathrm{NiF}_{4}$ ( $\left.\operatorname{cross}^{92,93}\right), \mathrm{Rb}_{2} \mathrm{Ni} \mathrm{F}_{4}$ (up triangle ${ }^{94}$ ), $\mathrm{Rb}_{2} \mathrm{MnCl}_{4}$ ( $\left.\operatorname{circle}^{95}\right), \mathrm{Rb}_{2} \mathrm{MnF}_{4}$ (diamond $\left.{ }^{92.96}\right)$. In the inset the region of weak anisotropy is enlarged.

important in view of the characterization of experimentally accessible materials, we verified that the Ising-like transition temperature decreases very slowly (logarithmically) towards its vanishing value in the isotropic limit, so that $t_{\mathrm{c}}$ remains substantially of the order of unity.

As a sample of the various results that were obtained, we report in Fig. 15 the comparison ${ }^{85}$ with the experimental data 97 for the correlation length of the $S=5 / 2$ magnet $\mathrm{Rb}_{2} \mathrm{MnF}_{4}$, that results quite well described by the anisotropic model with $J=7.42 \mathrm{~K}$ and $\mu=0.9942$. $\mathrm{Rb}_{2} \mathrm{MnF}_{4}$ is known to behave as a $2 \mathrm{D}$ magnet both above and below the observed transition ${ }^{76}$, so that the critical behavior is not contaminated by the onset of 3D order and a clean characterization of the transition is possible. In Ref. 85 we have compared our theoretical results also with the neutron scattering experimental data for the staggered magnetization, staggered susceptibility and correlation length of $\mathrm{Rb}_{2} \mathrm{MnF}_{4}$ and found an excellent agreement both for the overall temperature behavior and for the value of the critical temperature, that perfectly coincides with the one deriving from the experimental analysis, $T_{\mathrm{c}}=38.4 \mathrm{~K}$ (i.e., $t_{\mathrm{c}}=0.575$ ).

Another quantity that shows a signature of the anisotropy and of the Ising transition is the specific heat: in Fig. [16] a comparison with experimental data is shown in the case of the $S=5 / 2$ compound Mn-formate diUrea ${ }^{88}$, whose anisotropy can be estimated from the sole knowledge of the exchange integral and of the measured 


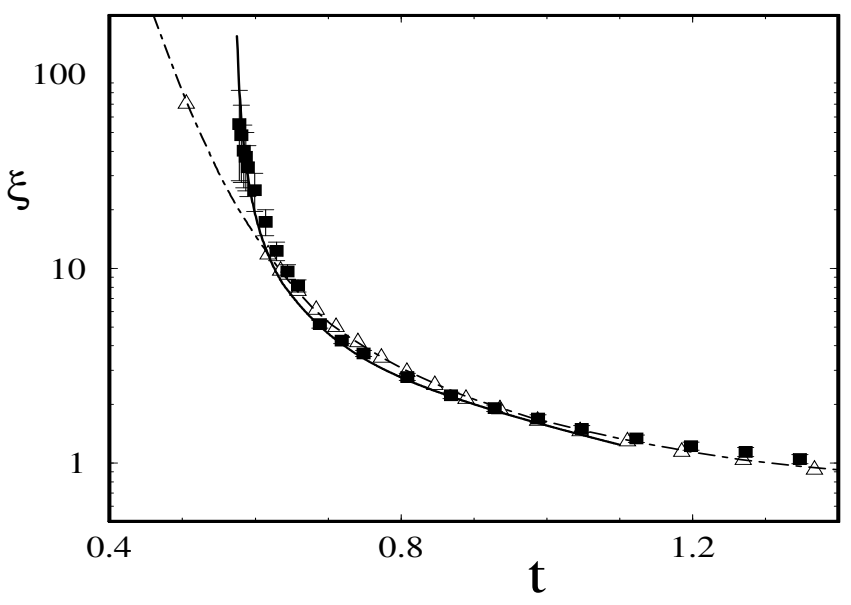

FIG. 15: Correlation length vs $t$ for $S=5 / 2, \mu=0.9942$ (full curve) and $\mu=1$ (isotropic, dash-dotted curve); the symbols are neutron scattering data ${ }^{97}$ for $\mathrm{Rb}_{2} \mathrm{MnF}_{4}$. The triangles are quantum Monte Carlo data ${ }^{98}$ for the isotropic model.

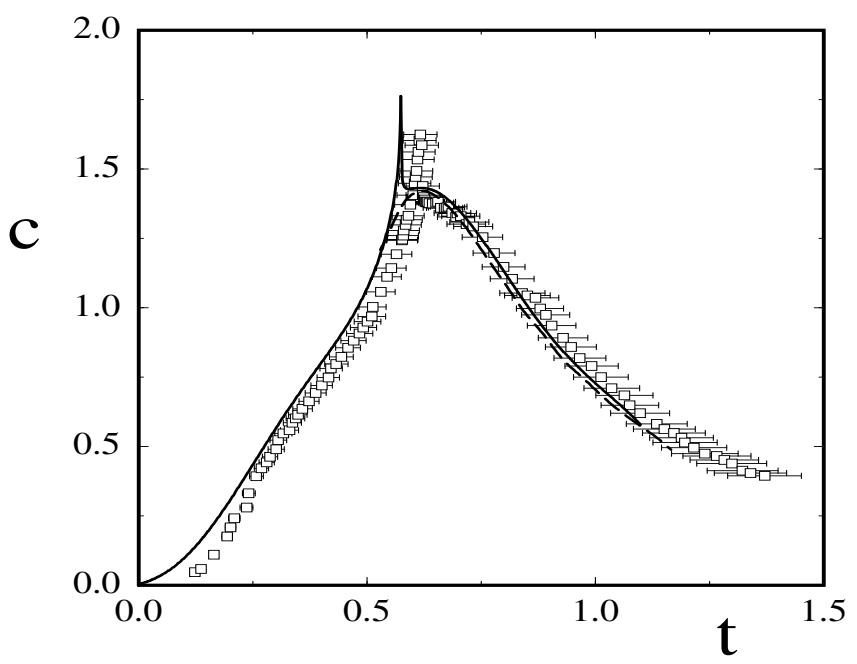

FIG. 16: Specific heat vs $t=T / J \widetilde{S}^{2}$, for $S=5 / 2$. Mnf-2U experiments (squares) ${ }^{100}$, EA-QHAF with $\mu=0.9942$ (solid line) ${ }^{87.88}$, isotropic QHAF (dashed line). Note that the correct anisotropy for this compound is estimated to be $\mu=0.981$. Error bars are due to the experimental uncertainty on $J$ for Mn-f-2U.

transition temperature to be $\mu=0.981$. The comparison reveals the existence of a crossover from a hightemperature 2D-Heisenberg regime to a critical 2D-Ising regime that triggers the observed ${ }^{99} 3 \mathrm{D}$ phase transition at $T_{\mathrm{N}}=3.77 \mathrm{~K}$.

Finally, for the strongest quantum case, $S=1 / 2$, we have used the continuous-time quantum Monte Carlo method based on the loop algorithm ${ }^{84}$. The general outcome of the numerical simulations is that the thermodynamics of $2 \mathrm{D}$ quantum antiferromagnets is extremely sensitive to the presence of weak easy-axis anisotropies of the order of those of real compounds. For instance, in Fig. 17 it is shown that for $\mu=0.99$ the uniform sus-

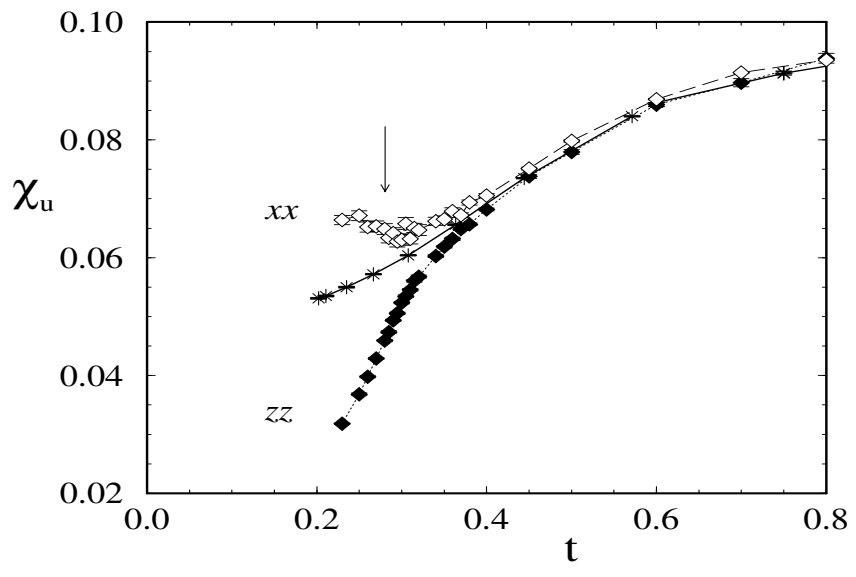

FIG. 17: Uniform susceptibility of the EA model for $\mu=0.99$ from quantum Monte Carlo simulations ${ }^{84}$. Full diamonds: longitudinal branch; open diamonds: transverse branch; stars: data for the isotropic mode $\mathrm{l}^{101}$. The lines are guides for the eye. The arrow indicates the estimated critical temperature.

ceptibility, which is a noncritical quantity, undoubtedly shows a characteristic anisotropic behavior with a different temperature dependence of the transverse and longitudinal branches: the former displays a minimum and the latter monotonically goes to zero, as expected for an EA antiferromagnet. This behavior results from the anisotropy-induced spin ordering, that makes the system more sensitive to the application of a transverse magnetic field, rather than of a longitudinal one. Both the minimum of the in-plane component and the decrease of the longitudinal one are close to the transition, a feature also peculiar to the Ising model. Results for the critical temperature at $S=1 / 2$ are already included in Fig. 14.

\section{B. 2D antiferromagnet with easy-plane anisotropy}

In the case of an easy-plane anisotropy the MerminWagner theorem still holds, so that no finite-temperature transition towards a phase with a finite order parameter may occur. However, a Berezinskii-Kosterlitz-Thouless (BKT) transition ${ }^{102.103}$, related with the existence of vortex-like topological excitations, is known to characterize the class of the easy-plane models and may occur at a critical temperature $t_{\mathrm{c}}(\lambda, S)>0$. The reference system for the easy-plane class is the planar rotator model, or $X Y$ model, defined in terms of two-component spins: above $t_{\mathrm{c}}$ the system is disordered, with exponentially decaying correlation functions; in the region $0<t<t_{\mathrm{c}}$ the system is in a critical phase with vanishing magnetization and power-law decaying correlators (quasi-long-range order); at $t=0$ the magnetization gets a finite value and the system is ordered.

The observation of clear signatures of BKT critical behavior in real magnets is a controversial issue. However, it can explain the properties of several layered compounds $53,104,105,106$ whose high-temperature phase can be 
described by a purely 2D Heisenberg Hamiltonian, with an exchange interaction often displaying weak easy-plane (EP) anisotropies, on the order of $10^{-2} \div 10^{-4}$ times the dominant isotropic coupling ${ }^{105.106}$. Symmetry and universality arguments suggest that the EP anisotropy drives the system towards a BKT behavior at finite temperature, and the enhanced intraplane correlations trigger the transition to the observed $3 \mathrm{D}$ ordered state. As a consequence, 2D critical behavior in close proximity of the would-be BKT transition is masked by these 3D effects.

The EP-QHAF Hamiltonian reads

$$
\mathcal{H}=\frac{J}{2} \sum_{\boldsymbol{i}, \boldsymbol{d}}\left[S_{\boldsymbol{i}}^{x} S_{\boldsymbol{i}+\boldsymbol{d}}^{x}+S_{\boldsymbol{i}}^{y} S_{\boldsymbol{i}+\boldsymbol{d}}^{y}+\lambda S_{\boldsymbol{i}}^{z} S_{\boldsymbol{i}+\boldsymbol{d}}^{z}\right]
$$

where $\lambda$ is the easy-plane anisotropy parameter $(0 \leq \lambda<1)$. Again, $J \widetilde{S}^{2} \equiv J(S+1 / 2)^{2}$ sets the overall energy scale and $t=T / J \widetilde{S}^{2}$ is the reduced temperature. When $\lambda=1$ the model reduces to the isotropic QHAF. Note that a canonical transformation reversing the $x$ and $y$ spin components one one sublattice is equivalent to setting $(J, \lambda) \rightarrow(-J,-\lambda)$, so that negative values of $\lambda(-1<\lambda \leq 0)$ correspond to the easy-plane ferromagnet. The $\lambda=0$ case is called $X Y$ model or XX0 model. However, at variance with the planar rotator model, out-of-plane fluctuations are present both in the classical and in the quantum EP models. Nevertheless, if $|\lambda|<1$, the classical EP model still undergoes a BKT phase transition ${ }^{107}$. MC simulations of the classical systems $108,109,110,111$ confirm that the planar and the XXZ model share the same qualitative behavior, but the value of the transition temperature of the planar mode ${ }^{112}$ lowers by $22 \%$ in the XX0 (i.e., with $\lambda=0$ ) model110.111, as a consequence of out-of-plane fluctuations. A renormalization group calculation ${ }^{107}$ predicts that the transition temperature vanishes logarithmically as the isotropic limit $|\lambda| \rightarrow 1$ is approached, and this was also verified in classical MC simulations ${ }^{110}$. Experiments 53.54 and quantum MC simulations ${ }^{113}$ indicated that the qualitative behavior of the BKT transition is preserved in the quantum system, with only quantitative modifications of the critical parameters due to the quantum fluctuations.

We applied the effective Hamiltonian formalism ${ }^{65,66}$ to the EP-QHAF, finding that it was necessary ${ }^{87,114}$ to resort to the Villain or to the Holstein-Primakoff transformation, depending on the anisotropy being strong or weak, respectively. While the above approach gives reliable results (with a smooth enough connection at intermediate anisotropy) for spin $S \geq 1$, we adopted quantum Monte Carlo simulations $84 . \overline{88} .115 .116 .117$ in the case $S=1 / 2$. The effective Hamiltonian 87.114 .118 for the EP$\mathrm{QHAF}$, in terms of classical spins, takes the form

$$
\frac{\mathcal{H}_{\mathrm{eff}}}{J \widetilde{S}^{2}}=\frac{j_{\mathrm{eff}}}{2} \sum_{\boldsymbol{i}, \boldsymbol{d}}\left[s_{\boldsymbol{i}}^{x} s_{\boldsymbol{i}+\boldsymbol{d}}^{x}+s_{\boldsymbol{i}}^{y} s_{\boldsymbol{i}+\boldsymbol{d}}^{y}+\lambda_{\mathrm{eff}} s_{\boldsymbol{i}}^{z} s_{\boldsymbol{i}+\boldsymbol{d}}^{z}\right]
$$

and displays a weaker renormalized exchange $j_{\text {eff }}(t, \lambda)<J$ and easy-plane anisotropy $\lambda_{\text {eff }}(t, \lambda)>\lambda$

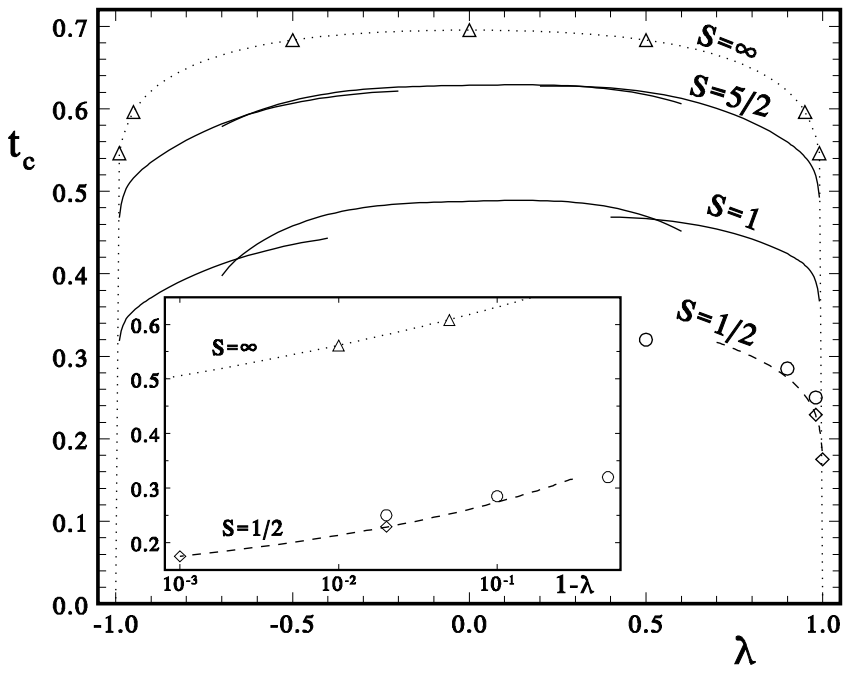

FIG. 18: BKT critical temperature vs anisotropy $\lambda$ for the easy-plane model with $S=1 / 2,1,5 / 2, \infty$. The triangles report the classical $(S=\infty)$ MC data 110 used to construct the curve for $t_{\mathrm{c}}^{\mathrm{cl}}(\lambda)$ and hence the renormalized curves for $S=1$ and 5/2: the curves obtained through the Holstein-Primakoff $(\lambda \lesssim 0.5)$ and the Villain $(\lambda \gtrsim 0.5)$ spin-boson transformation are seen to connect in a fairly smooth way. The diamonds are our QMC data ${ }^{84}$ for $S=1 / 2$, while the circles are earlier QMC results ${ }^{113}$. The inset expands the nearly isotropic region, in which the expected logarithmic behavior $t_{\mathrm{c}}(\lambda) \sim(a-\ln |1-\lambda|)^{-1}$ is fitted by the dashed curve.

(an additional free-energy term is not reported). In analogy to the EA case, the BKT transition temperature can be obtained by renormalization of the classical one using the self-consistent relation

$$
t_{\mathrm{c}}(\lambda, S)=j_{\mathrm{eff}}(t, \lambda, S) t_{\mathrm{c}}^{\mathrm{cl}}\left(\lambda_{\mathrm{eff}}(t, \lambda, S)\right) .
$$

In Fig. 18 the phase diagram of the EP-QHAF is reported, including the QMC results for $S=1 / 2$. It is seen that the BKT transition temperature stays large (i.e., comparable to the exchange constant) also for very weak EP anisotropy.

However, as explained above, the problem of detecting the incipient BKT transition requires to look for signatures of XY behavior in a region above the transition. We have shown 115 that a suitable quantity is the uniform susceptibility $\chi_{\mathrm{u}}^{\alpha \alpha}$, which has in-plane $(\alpha=x, y)$ and out-ofplane $(\alpha=z)$ components and is noncritical, i.e., it does not show singularities at $t_{\mathrm{c}}$. Fig. [19] shows indeed that $\chi_{\mathrm{u}}^{z z}$ deviates from the isotropic $\chi_{\mathrm{u}}$ and displays a minimum. A similar feature is also present in other quantities 84,115 and occurs around the temperature $t_{\mathrm{co}}(\lambda)$ that can be generally defined as the minimum of $\chi_{\mathrm{u}}^{z z}(t, \lambda)$. The pronounced deviation of $\chi_{\mathrm{u}}^{z z}$ from the isotropic behavior is due to a simple statistical reason. The uniform susceptibility arises from spin canting: two antiferromagnetically coupled spins in an infinitesimal magnetic field $\boldsymbol{h}$ minimize their energy when they lie almost orthogonal to $\boldsymbol{h}$ and slightly cant in its direction, thus giving a linear response; if they are locally parallel to $\boldsymbol{h}$ the response is 


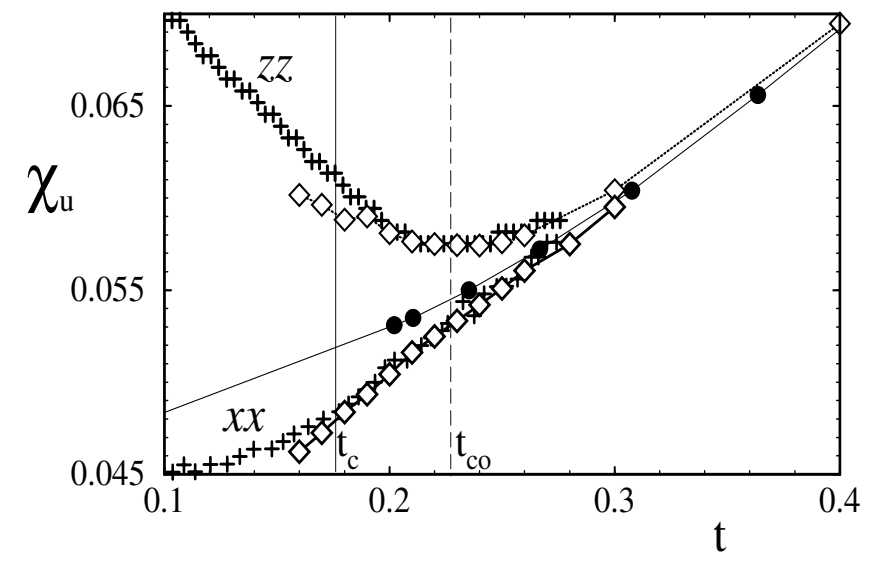

FIG. 19: Out-of-plane $(z z)$ and in-plane $(x x)$ uniform susceptibility $\chi_{\mathrm{u}}$ for $\lambda=0.999$. Diamonds are our QMC results, crosses are experimental data ${ }^{119,120}$ for $\mathrm{Sr}_{2} \mathrm{CuO}_{2} \mathrm{Cl}_{2}$. The circles report the result for the isotropic QHAF. The vertical lines mark the $3 \mathrm{D}$ transition temperature $t_{\mathrm{N}}=0.176$ and the crossover temperature $t_{\mathrm{co}} \simeq 0.227$.

instead negligible. When for $t \lesssim t_{\text {co }}$ the anisotropy becomes effective, the fraction of spins aligned in the EP rapidly increases compared to that of the isotropic case $(\sim 2 / 3)$ and the response to a field along $z$ (i.e., $\left.\chi_{\mathrm{u}}^{z z}\right)$ is proportionally larger.

The layered cuprate $\mathrm{Sr}_{2} \mathrm{CuO}_{2} \mathrm{Cl}_{2}$ is a good realization $^{53}$ of the EP-QHAF model, with $J=1450 \mathrm{~K}$; considering the spin-wave gap renormalization, its bare anisotropy is estimated to be $\Delta \simeq 0.0014$. Experimental data for the uniform susceptibility of this compound ${ }^{119.120}$ ) are reported in Fig. 19. They excellently compare with our results at $\lambda=0.999$ : the position of the minimum of $\chi_{\mathrm{u}}^{z z}$ gives $t_{\mathrm{co}}=0.227(15)$. Close to the critical region the experimental data are affected by the $3 \mathrm{D}$ nature of the ordering of the real magnet: the Néel transition is observed at $t_{\mathrm{N}}=0.176(10)$ and well compares with the theoretical estimate $t_{\mathrm{c}}(\lambda=0.0014)=0.179(10)$, confirming that $3 \mathrm{D}$ ordering is induced by the incipient intra-layer BKT transition.

It is worthwhile to mention that on the triangular lattice the easy-plane antiferromagnet has very peculiar behavior, already at the classical level, due to the frustration effect of accommodating three antiferromagnetic spins on a plaquette. Indeed the minimum energy corresponds to a configuration with the three sublattices aligned in the easy plane at equal angles $2 \pi / 3$. As clockwise and counterclockwise plaquette vorticities are possible, this configuration is twofold degenerate and there is chiral symmetry, which corresponds to an Ising-like order parameter. Therefore both a BKT and an Ising transition coexist in the system. We have studied the triangular antiferromagnet both in the classical ${ }^{121}$ and in the quantum ${ }^{122}$ case, constructing the phase diagram for varying anisotropy and showing that the transitions occur at slightly different temperatures.

\section{2D antiferromagnet in an applied Zeeman field}

An interesting behavior is shown by the 2D Heisenberg antiferromagnet when a magnetic field is applied. Indeed, a frustration phenomenon occurs, as antiferromagnetism tends to antialign spins while the field tends to align them with itself: in the classical system ${ }^{123}$ it appears that the minimum-energy configuration is the one with the spins almost orthogonal to the field and canted in its direction. Therefore, provided the field is not strong enough to overcome the exchange and to saturate the magnetization, it acts as an effective easy-plane anisotropy and one expects to observe BKT behavior. Remarkably, as this can be induced in a real quasi-2D antiferromagnetic system by means of an applied field, the strength of the effective anisotropy is in this case tunable. Even though $2 \mathrm{D}$ criticality just acts as a trigger for $3 \mathrm{D}$ ordering, by observing that the critical temperature follows the predicted behavior upon the field, an experiment could realize an objective observation of genuine 2D behavior.

The 2D QHAF in a uniform magnetic field is described by the Hamiltonian

$$
\mathcal{H}=\frac{J}{2} \sum_{\boldsymbol{i}, \boldsymbol{d}} \boldsymbol{S}_{\boldsymbol{i}} \cdot \boldsymbol{S}_{\boldsymbol{i}+\boldsymbol{d}}-g \mu_{\mathrm{B}} H \sum_{\boldsymbol{i}} S_{\boldsymbol{i}}^{z}
$$

where $H$ is the applied Zeeman field, $\mu_{\mathrm{B}}$ the Bohr magneton, and $g$ the gyromagnetic ratio.

We have studied ${ }^{124,125}$ the $S=\frac{1}{2} 2 \mathrm{D}$ QHAF in an uniform magnetic field by means of the QMC method based on the worm algorithm. Our results confirmed that an arbitrarily small field is able to induce a BKT transition and an extended $X Y$ phase above it, as in the case of an easy-plane exchange anisotropy. The field-induced $X Y$ behavior becomes more and more marked for increasing fields, while for strong fields the antiferromagnetic behavior along the field axis is nearly washed out, so that the system behaves as a planar rotator model with antiferromagnetism surviving in the orthogonal plane only; the BKT critical temperature, as reported in Fig 20 (where $t \equiv T / J$ and $\left.h \equiv 2 g \mu_{\mathrm{B}} H / J\right)$, vanishes as the field reaches the saturation value $h_{\mathrm{c}}$ and the effective rotator length goes to zero. We have therefore shown that the model in a moderately strong field represents an ideal realization of the $X Y$ model and that $X Y$ behavior can be detected by measuring standard non-critical quantities, as the specific heat or the induced magnetization; an experimental realization of the $X Y$ model in purely magnetic systems and a systematic investigation of the dynamics of vortex/antivortex excitations is therefore possible.

\section{FRUSTRATION IN THE 2D QUANTUM $J_{1}-J_{2}$ HEISENBERG MODEL}

The study of frustrated quantum spin systems is one of the most challenging and exciting topics in theoretical magnetism because of the possible existence of a non 


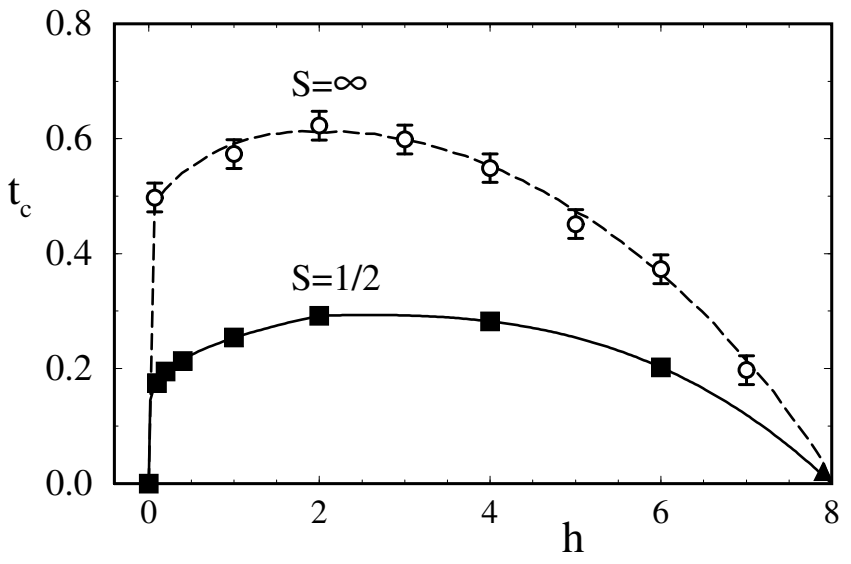

FIG. 20: Phase diagram of the $S=\frac{1}{2} 2 \mathrm{D}$ QHAF in a magnetic field. Open symbols refer to the classical limit of the mode ${ }^{123}$; the triangle ${ }^{126}$ and the squares ${ }^{124}$ are QMC results.

magnetic zero temperature phase. A very extensively investigated, yet largely debated model is the so-called $J_{1}-J_{2}$ Heisenberg model with competing antiferromagnetic couplings $\left(J_{1}, J_{2}>0\right)$ between nearest-neighbors (nn) and next-nearest-neighbors (nnn)

$$
\mathcal{H}=J_{1} \sum_{\mathrm{nn}} \boldsymbol{S}_{\boldsymbol{i}} \cdot \boldsymbol{S}_{\boldsymbol{j}}+J_{2} \sum_{\mathrm{nnn}} \boldsymbol{S}_{\boldsymbol{i}} \cdot \boldsymbol{S}_{\boldsymbol{j}}
$$

where the spin operators are defined on a periodic lattice with $N=L \times L$ sites; hereafter $\alpha=J_{2} / J_{1}$ defines the frustration ratio.

In the classical limit $(S \rightarrow \infty)$, the minimum energy configuration has conventional Néel order with magnetic wave vector $\boldsymbol{Q}=(\pi, \pi)$ for $\alpha<0.5$. Instead, for $\alpha>0.5$, the antiferromagnetic order is established independently on the two sublattices, with the two staggered magnetizations free to rotate with respect to each other. One of the two families of collinear states, with pitch vectors $\boldsymbol{Q}=(\pi, 0)$ or $(0, \pi)$, are selected by an order-by-disorder mechanism as soon as thermal or quantum fluctuations are taken into account. As a result, for $\alpha>0.5$ the classical ground state breaks not only the spin rotational and translational invariance of the Hamiltonian - as the conventional Néel phase - but also its invariance under $\pi / 2$ lattice rotations, the resulting degeneracy corresponding to the group $O(3) \times Z_{2}$. Remarkably, the additional discrete $Z_{2}$ symmetry can in principle be broken at finite temperatures without violating the Mermin-Wagner theorem. On this basis, in a seminal paper ${ }^{127}$, Chandra, Coleman, and Larkin (CCL) proposed that the 2D $J_{1}-J_{2}$ model could sustain an Ising phase transition at finitetemperature, with an order parameter directly related to the $Z_{2}$ degree of freedom induced by frustration. They also provided quantitative estimates of the critical temperatures in the large- $\alpha$ limit for both the classical and the quantum cases.

This transition in the classical model has been established by an extensive Monte Carlo $\stackrel{128}{2}$. In the quantum case, the occurrence of a low-temperature phase with a discrete broken symmetry has been subject of debates in connection with the discovery of three vanadate compounds $\left(\mathrm{Li}_{2} \mathrm{VOSiO}_{4}, \mathrm{Li}_{2} \mathrm{VOGeO}_{4}\right.$, and $\left.\mathrm{VOMoO}_{4}\right)$ whose relevant magnetic interactions involve nearest and nextnearest spin-1/2 $V^{4+}$ ions on weakly coupled stacked planes. In particular, NMR and $\mu \mathrm{SR}$ measurements on $\mathrm{Li}_{2} \mathrm{VOSiO}_{4}{ }^{129}$ indicate the occurrence of a transition to a low-temperature phase with collinear order at $T_{\mathrm{N}} \simeq 2.8 \mathrm{~K}$. However in the experiments with vanadate compounds, structural distortions, interlayer and anisotropy effects are likely to come into play ${ }^{130}$, and on the other hand the theoretical investigation cannot rely on the insight provided by quantum Monte Carlo methods as their reliability in presence of frustration is strongly limited (see the review articles in Ref. 131 132).

A complete study of the thermodynamic properties of the the quantum $J_{1}-J_{2}$ model in its collinear phase has been pursued within the PQSCHA scheme ${ }^{65}$ described in Section IV by which the thermodynamics is rephrased in terms of a classical effective Hamiltonian with renormalized parameters depending on the spin value, temperature, and frustration. It is possible to show that, to $O(1 / S)$, the effective Hamiltonian can be recast in a form preserving all the symmetries of the original model, and that reads (except for uniform terms):

$$
\mathcal{H}^{\mathrm{eff}}=J_{1}^{\mathrm{eff}} \widetilde{S}^{2} \sum_{\mathrm{nn}} s_{i} \cdot s_{j}+J_{2}^{\mathrm{eff}} \widetilde{S}^{2} \sum_{\mathrm{nnn}} s_{i} \cdot s_{j}
$$

where $s_{i}$ are classical vectors of length $1, \widetilde{S}=S+\frac{1}{2}$ is the effective spin length, and $J_{1}^{\text {eff }}=\left(\theta_{x}^{2}+\theta_{y}^{2}\right) \theta_{2}^{2} J_{1} / 2$, $J_{2}^{\text {eff }}=\theta_{2}^{4} J_{2}$, are the quantum-renormalized exchange integrals, with spin-, temperature- and frustrationdependent renormalization parameters $\theta_{x}, \theta_{y}$ and $\theta_{2}$.

The occurrence of the transition ${ }^{127}$ in the quantum case can be directly addressed within our approach by calculating the critical temperatures as functions of the spin and of the frustration ratio $\frac{133}{}$. Using a simple scaling argument the critical temperatures in the quantum case $T_{\mathrm{c}}(S, \alpha)$ can be related to those of the classical model $T_{\mathrm{c}}^{(c l)}(\alpha)$ through the following self-consistent relation 87.122

$$
T_{\mathrm{c}}(S, \alpha)=j_{1}^{\mathrm{eff}}\left(T_{\mathrm{c}}, S, \alpha\right) T_{\mathrm{c}}^{(c l)}\left(\alpha^{\mathrm{eff}}\left(T_{\mathrm{c}}, S, \alpha\right)\right),
$$

where $j_{1}^{\text {eff }}=J_{1}^{\text {eff }} \widetilde{S}^{2} / J_{1}$ and $\alpha^{\text {eff }}=J_{2}^{\text {eff }} / J_{1}^{\text {eff }}$. The classical transition temperature, $T_{\mathrm{c}}^{(c l)}(\alpha)$ is accurately known through extensive MC simulations for $\alpha \leq 2$; it vanishes for $\alpha \rightarrow 1 / 2$ and grows more or less linearly for $\alpha>1$.

The behavior of the transition temperature versus the frustration ration is plotted in Fig. 21 for different values of the spin length. In order to represent the whole interval of $\alpha \in[1 / 2, \infty)$ in Fig. 21 we have plotted both the MC and the CCL estimates of the classical critical temperatures as a function of $\alpha /(1+\alpha)$. The mismatch between the MC and CCL predictions is a minor flaw that can be corrected by slightly modifying CCL's criterion for the determination of the transition temperature 


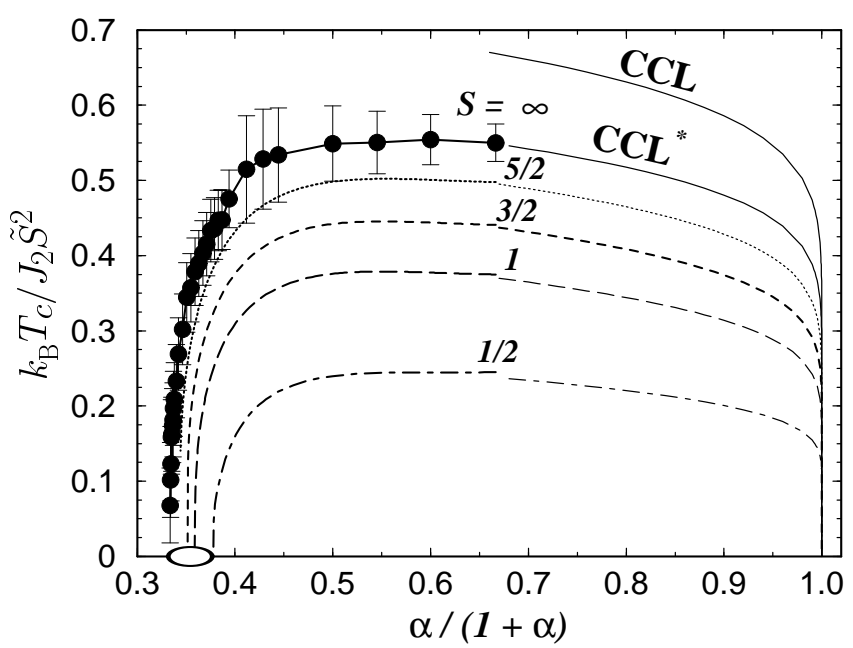

FIG. 21: Renormalized critical temperature of the CCL transition for various values of the spin. Classical data $(\bullet)$ are taken from Ref. 128. The solid lines on the right are the CCL and the CCL* prediction for the classical and $S=1 / 2$ case (see text). The ellipse on the horizontal axis mark the nonmagnetic (spin-liquid) phase between $\alpha_{\mathrm{c}}(S=\infty)=0.5$ and $\alpha_{\mathrm{c}}(S=1 / 2) \simeq 0.6$.

as explained in Ref. 128. Remarkably, while for large $\alpha$ the transition temperature vanishes for $\alpha \rightarrow \infty$ for any value of the spin, in the opposite limit the critical temperatures vanish approaching a critical value $\alpha_{\mathrm{c}}>0.5$ that increases as $S$ decreases, thus confirming the existence of a non-magnetic phase in the regime of high frustration. In particular for $S=1 / 2, \alpha_{\mathrm{c}} \simeq 0.6$ in agreement with the previous estimates of the zero-temperature quantum critical point ${ }^{132}$.

\section{CONCLUSIONS}

The activity in magnetism of the Condensed Matter Theory group in Firenze 134 stems from the early work on two-magnon Raman scattering in the seventies, and has grown up in the years with the collaboration of several scientists. In this paper we summarize the relevant theoretical work that concerns antiferromagnetic models. This activity has been mainly concentrated on lowdimensional systems and has found one of its main motivations in the intent of interpreting the data collected in experiments on real materials. Among the prominent subjects, we reported about soliton-excitation effects in one-dimensional systems, critical and near-to-critical behaviors and phase transitions in two-dimensional ones. Besides that, we also faced some intriguing theoretical problems where fundamental aspects of Quantum Mechanics come into play, as, e.g., the ground state of antiferromagnetic chains with integer spin or the possible quantum critical regime predicted from the fieldtheory treatment of the two-dimensional antiferromagnetic Heisenberg model.
1 F. D. M. Haldane, Phys. Rev. Lett. 50, 1153 (1983); Phys. Lett. A 93, 464 (1983).

2 A. Sokol and D. Pines, Phys. Rev. Lett. 71, 2813 (1993).

3 S. Chakravarty, in High Temperature Superconductivity, eds. K. S. Bedell, D. Coffey, D. E. Meltzer, D. Pines, and J. R. Schrieffer (Addison Wesley, Redwood City, 1990).

4 N. D. Mermin and H. Wagner, Phys. Rev. Lett. 17, 1133 (1966).

5 P. A. Fleury, Int. J. Magnetism, 1, 75 (1970).

${ }^{6}$ R. Loudon, Adv. Phys. 17, 243 (1968); M. G. Cottam and D. J. Lockwood Light scattering in magnetic solids (Wiley, New York, 1986).

7 P. A. Fleury, Phys. Rev., 180, 591 (1969).

${ }^{8}$ U. Balucani and V. Tognetti, Riv. N. Cim. 6, 39 (1976).

${ }^{9}$ R. J. Elliott and M. Thorpe, J. Phys. C 2, 1630 (1969).

10 U. Balucani and V. Tognetti, Phys. Rev. B 8, 4247 (1973).

11 U. Balucani, M. H. Lee, and V. Tognetti, Phys. Rep. 373, 409 (2003).

12 F. Barocchi, P. Mazzinghi, V. Tognetti, and M. Zoppi, Sol. St. Comm. 25, 241 (1978).

13 S. R. Chinn, R. W. Davies, and H. J. Zeiger, AIP Conf. Proc. 5, 317 (1972)

14 H. Mori, Progr. Theor. Phys. 33, 423 (1965); 34, 399 (1965).

15 U. Balucani and V. Tognetti, Phys. Rev. B 16, 271 (1977).

16 U. Balucani, M. G. Pini, and V. Tognetti, N. Cim. B 46, 81 (1978).
17 V. E. Korepin, N. M. Bogoliubov, and A. G. Izergin, Quantum inverse scattering method and correlation functions (Cambridge Univ. Press, 1993).

18 H. J. Mikeska, J.Phys. C 11, L29 (1978); 13, 2913 (1980).

19 F. Currie, J. A. Krumhansl, A. R. Bishop, and S. E. Trullinger, Phys. Rev. B 22, 477 (1980).

20 T. Schneider and E. Stoll, Phys. Rev. B 22, 5317 (1980).

21 F. Borsa, M. G. Pini, A. Rettori, and V.Tognetti, Phys. Rev. B 28, 5173 (1983).

22 Magnetic Excitations and Fluctuations, eds. S. W. Lovesey, U. Balucani, F. Borsa, and V. Tognetti, Springer Series in Solid State 54 (Springer Verlag, Berlin, 1984).

${ }^{23}$ E. H. Lieb, T. D. Schultz, and D. C. Mattis, Ann. Phys. (New York) 16, 407 (1961).

24 I. Affleck, T. Kennedy, E. H. Lieb, and H. Tasaki, Phys. Rev. Lett. 59, 799 (1987); Commun. Math. Phys. 115, 477 (1988).

25 D. P. Arovas, A. Auerbach, F. D. M. Haldane, Phys. Rev. Lett. 60, 531 (1988).

${ }^{26}$ I. Affleck, J. Phys. Condens. Matter 1, 3407 (1989).

27 M. Takahashi, Phys. Rev. Lett. 62, 2313 (1989).

28 W. J. L. Buyers, R. M. Morra, R. L. Armstrong, M. J. Hogan, P. Gerlach, and K. Hirakawa, Phys. Rev. Lett. 56, 371 (1986).

${ }^{29}$ M. Steiner, K. Kakurai, J. K. Kjems, D. Petitgrand, and R. Pynn, J. Appl. Phys. 61, 3953 (1987).

30 M. Date and K. Kindo, Phys. Rev. Lett. 63, 1424 (1990). 
31 P. Gaveau, J. P. Boucher, L. P. Regnault, and J. P. Renard, Europhys. Lett. 12, 647 (1990).

32 L. C. Brunel, T. Brill, I. Zaliznyak, J. P. Boucher, and J. P. Renard, Phys. Rev. Lett. 69, 1699 (1992).

33 R. Botet, R. Jullien, and M. Kolb, Phys. Rev. B 28, 3914 (1983).

34 J. B. Parkinson and J. C. Bonner, Phys. Rev. B 32, 4703 (1985).

35 M. P. Nightingale and H. W. J. Blöte, Phys. Rev. B 33, 659 (1986)

36 T. Kennedy, J. Phys. Condens. Matter 2, 5737 (1990).

37 T. Sakai and M. Takahashi, Phys. Rev B 42, 1090 (1990).

38 T. Delica, K. Kopinga, H. Leschke, and K. K. Mon, Europhys. Lett. 15, 55 (1991).

39 H. Koehler and R. Shilling, J. Phys. Condens. Matter 4, 7899 (1992).

40 H.-J. Mikeska, Chaos, Solitons and Fractals 5, 2585 (1995).

41 H.-J. Mikeska, Europhys. Lett. 19, 39 (1992).

42 G. Gomez-Santos, Phys. Rev. Lett. 63, 790 (1989).

43 T. Kennedy and H. Tasaki, Phys. Rev. B 45, 304 (1992); Commun. Math. Phys. 147, 431 (1992).

44 M. den Nijs and K. Rommelse, Phys. Rev. B 50, 3907 (1994).

45 H. Tasaki, Phys. Rev. Lett. 66, 798 (1991).

46 H.-J. Mikeska and P. Verrucchi, Phys. Rev. B 52, 3571 (1995).

47 O. Golinelli, Th. Joliceur, and R. Lacaze, Phys. Rev. B 50, 3037 (1994).

48 N. Elstner and H.-J. Mikeska, Phys. Rev. B 50, 3907 (1994).

49 E. J. Neves and J. F. Perez, Phys. Lett. 114A, 331 (1986).

50 E. Manousakis, Rev. Mod. Phys. 63, 1 (1991).

${ }^{51}$ M. A. Kastner, R. J. Birgeneau, G. Shirane, and Y. Endoh, Rev. Mod. Phys. 70, 897 (1998)

52 M. Greven, R. J. Birgeneau, Y. Endoh, M. A. Kastner, B. Keimer, M. Matsuda, G. Shirane, and T. R. Thurston, Phys. Rev. Lett. 72, 1096 (1994).

${ }^{53}$ M. Greven, R. J. Birgeneau, Y. Endoh, M. A. Kastner, M. Matsuda, and G. Shirane, Z. Phys. B 96, 465 (1995).

54 K. Nakajima, K. Yamada, S. Hosoya, Y. Endoh, M. Greven, and R. J. Birgeneau, Z. Phys. B 96, 479 (1995).

55 Y. S. Lee, M. Greven, B. O. Wells, R. J. Birgeneau, and G. Shirane, Eur. Phys. J. B 5, 15 (1998).

56 S. Fulton, S. E. Nagler, L. M. N. Needham, and B. M. Wanklyn, J. Phys.: Condens. Matter 6, 6667 (1994); S. Fulton, R. A. Cowley, A. Desert, and T. Mason, J. Phys. Condens. Matter 6, 6679 (1994).

57 P. Carretta, T. Ciabattoni, A. Cuccoli, E. Mognaschi, A. Rigamonti, V. Tognetti, and P. Verrucchi, Phys. Rev. Lett. 84, 366 (2000).

58 S. Chakravarty, B. I. Halperin, and D. R. Nelson, Phys. Rev. B 39, 2344 (1989).

59 I. Affleck, Nucl. Phys. B257, 397 (1985).

60 P. Hasenfratz and F. Niedermayer, Phys. Lett. B 268, 231 (1991).

61 N. Elstner, A. Sokol, R. R. P. Singh, M. Greven, and R. J. Birgeneau, Phys. Rev. Lett. 75, 938 (1995); N. Elstner, Int. J. Mod. Phys. B 11, 1753 (1997).

62 A. Cuccoli, V. Tognetti, P. Verrucchi, and R. Vaia, Phys. Rev. Lett. 77, 3439 (1996).

63 A. Cuccoli, V. Tognetti, P. Verrucchi, and R. Vaia, Phys. Rev. B 56, 14456 (1997).

64 A. Cuccoli, V. Tognetti, P. Verrucchi, and R. Vaia, Phys.
Rev. B 58, 14151 (1998).

65 A. Cuccoli, R. Giachetti, V. Tognetti, R. Vaia, and P. Verrucchi, J. Phys.: Condens. Matter 7, 7891 (1995).

66 A. Cuccoli, V. Tognetti, P. Verrucchi, and R. Vaia, Phys. Rev. A 45, 8418 (1992).

67 B.B. Beard, A. Cuccoli, R. Vaia, and P. Verrucchi, Phys. Rev. B 68, 104406 (2003).

68 P. Hasenfratz, Eur. Phys. J. B 13, 11 (2000).

69 M. Falcioni and A. Treves, Nucl. Phys. B265, 671 (1986); S. Caracciolo and A. Pelissetto, Nucl. Phys. B 420, 141 (1994).

70 P. Carretta, A. Rigamonti, and R. Sala, Phys. Rev. B 55, 3734 (1997)

71 J.-K. Kim, D. P. Landau, and M. Troyer, Phys. Rev. Lett. 79, 1583 (1997); private communication.

72 M. S. Makivic̀ and H.-Q. Ding, Phys. Rev. B 43, 3562 (1991).

73 B. B. Beard, R. J. Birgeneau, M. Greven, and U.-J. Wiese, Phys. Rev. Lett. 80, 1742 (1998). B. B. Beard, Nucl. Phys. Proc. Suppl. 83-84, 682 (2000).

${ }^{74}$ K. Harada, M. Troyer, and N. Kawashima, J. Phys. Soc. Jap. 67, 1130 (1998).

75 J. Kanamori, in Magnetism vol. 1, eds. G. T. Rado e H. Suhl (Academic Press, New York, 1963).

76 R. J. Birgeneau, H. J. Guggenheim, G. Shirane, Phys. Rev. B 1, 2211 (1970).

77 R .J. Birgeneau, H .J. Guggenheim, and G. Shirane, Phys. Rev. B 8, 304 (1973).

78 L. Onsager, Phys. Rev. 65, 117 (1944).

79 M. Bander and D. L. Mills, Phys. Rev. B 38, 12015 (1988).

80 J. D. Patterson and G. L. Jones, Phys. Rev. B 3, 131 (1971).

81 K. Binder and L. P. Landau, Phys. Rev. B 13, 1140 (1976).

82 P. A. Serena, N. García, and A. Levanyuk, Phys. Rev. B 47, 5027 (1993).

${ }^{83}$ M. E. Gouvêa, G. M. Wysin, S. A. Leonel, A. S. T. Pires, T. Kamppeter, and F. G. Mertens, Phys. Rev. B 59, 6229 (1999).

84 A. Cuccoli, T. Roscilde, V. Tognetti, P. Verrucchi, and R. Vaia, Phys. Rev. B 67, 104414 (2003).

85 A. Cuccoli, V. Tognetti, T. Roscilde, P. Verrucchi, and R. Vaia, Phys. Rev. B 62, 3771 (2000).

86 A. Cuccoli, T. Roscilde, V. Tognetti, R. Vaia, and P. Verrucchi, J. Magn. Magn. Mater. 226-230, 562 (2001).

87 A. Cuccoli, T. Roscilde, V. Tognetti, R. Vaia, and P. Verrucchi, Eur. Phys. J. B 20, 55 (2001).

88 A. Cuccoli, T. Roscilde, V. Tognetti, R. Vaia, and P. Verrucchi, J. Appl. Phys. 93, 7637 (2003).

89 A. Cuccoli, V. Tognetti, T. Roscilde, P. Verrucchi, and R. Vaia, Braz. J. Phys. 30, 697 (2000).

90 H.-Q. Ding, J. Phys.: Condens. Matter 2, 7979 (1990).

91 J. Rossat-Mignod, L. P. Regnault, C. Vettier, P. Burlet, J.Y. Henry, and G. Lapertot, Physica B 169, 58 (1991).

${ }^{92}$ H .W. de Wijn, L. R. Walker, and R. E. Walstedt, Phys. Rev. B 8, 285, (1973).

93 J. Skalyo, Jr., G. Shirane, R. J. Birgeneau, and H. J. Guggenheim, Phys. Rev. Lett. 23, 1394 (1969).

${ }^{94}$ K. Nagata and Y. Tomono, J. Phys. Soc. Japan 36, 78 (1974).

95 B. Schröder, V. Wagner, N. Lehner, K.A.M. Kesharwani, and R. Geick, Phys. Stat. Sol. (b) 97, 501 (1980).

96 R. A. Cowley, G. Shirane, R. J. Birgeneau, and H. J. 
Guggenheim, Phys. Rev. B 15, 4292 (1977).

97 Y. S. Lee, M. Greven, B. O. Wells, R. J. Birgeneau, and G. Shirane, Eur. Phys. J. B 5, 15 (1998).

98 B. B. Beard, Nucl. Phys. B, Proc. Suppl. 83-84, 682 (2000).

99 K. Takeda, H. Deguchi, T. Hoshiko, and K. Yamagata, J. Phys. Soc. Jap. 58, 3489 (1989).

100 K. Takeda, M. Mito, K. Nakajima, K. Kakurai, and K. Yamagata, Phys. Rev. B 63, 024425 (2001).

101 J.-K. Kim and M. Troyer, Phys. Rev. Lett. 80, 2705 (1998).

102 V. L. Berezinskii, Zh. Eksp. Teor. Fiz. 59, 907 (1970) [JETP 32, 493 (1971)].

103 J. M. Kosterlitz and D. J. Thouless, J. Phys. C 6, 1181 (1973); J. M. Kosterlitz, J. Phys. C 7, 1046 (1974).

104 B. J. Suh, F. Borsa, L. L. Miller, M. Corti, D. C. Johnston, and D. R. Torgeson, Phys. Rev. Lett. 75, 2212 (1995).

105 See, e.g.: Magnetic properties of layered transition metal compounds, ed. L. J. de Jongh (Kluwer, Dordrecht, 1990).

106 D. C. Johnston, in Handbook of magnetic materials, vol. 10, ed. K. H. J. Buschow (Elsevier Science, New York, 1997).

107 S. B. Khokhlachev, Zh. Exp. Teor. Fiz. 70, 265 (1976).

108 C. Kawabata and A. R. Bishop, Sol. St. Comm. 42, 595 (1982).

109 R. W. Gerling and D. P. Landau, in Magnetic Excitations and Fluctuations, eds. S. W. Lovesey, U. Balucani, F. Borsa, and V. Tognetti (Spinger Verlag, Berlin, 1984).

110 A. Cuccoli, V. Tognetti, and R. Vaia, Phys. Rev. B 52, 10221 (1995).

111 H. G. Evertz and D. P. Landau, Phys. Rev. B 54, 12302 (1996).

112 R. Gupta and C. Baillie, Phys. Rev. B 45, 2883 (1992).

113 H. Q. Ding and M. S. Makivić, Phys. Rev. B 42, 6827 (1990) and 45, 491 (1992); H.-Q. Ding, Phys. Rev. Lett. 68, 1927 (1992); Phys. Rev. B 45, 230 (1992).

114 L. Capriotti, A. Cuccoli, V. Tognetti, R. Vaia, and P. Verrucchi, Physica D 119, 68 (1998)

115 A. Cuccoli, T. Roscilde, R. Vaia, and P. Verrucchi, Phys. Rev. Lett. 90, 167205 (2003).
116 A. Cuccoli, T. Roscilde, V. Tognetti, R. Vaia, and P. Verrucchi, J. Appl. Phys. 93, 7640 (2003).

117 A. Cuccoli, T. Roscilde, V. Tognetti, R. Vaia, and P. Verrucchi, J. Magn. Magn. Mater. 272-276, e651 (2004).

118 A. Cuccoli, V. Tognetti, P. Verrucchi, and R. Vaia, Phys. Rev. B 51, 12840 (1995).

119 D. Vaknin, L. L. Miller, J. L. Zaretsky, and D. C. Johnston, Physica C 274, 331 (1997).

120 D. Vaknin, S. K. Sinha, C. Stassis, L. L. Miller, and D. C. Johnston, Phys. Rev. B 41, 1926 (1990).

121 L. Capriotti, R. Vaia, A. Cuccoli, and V. Tognetti, Phys. Rev. B 58, 273 (1998).

122 L. Capriotti, A. Cuccoli, V. Tognetti, P. Verrucchi, and R. Vaia, Phys. Rev. B 60, 7299 (1999).

123 D. P. Landau and K. Binder, Phys. Rev. B 24, 1391 (1981).

124 A. Cuccoli, T. Roscilde, R. Vaia, and P. Verrucchi, Phys. Rev. B 68, 060402 (2003).

125 A. Cuccoli, T. Roscilde, R. Vaia, and P. Verrucchi, J. Magn. Magn. Mater. 272-276, 884 (2004).

126 O. F. Syljuåsen, Phys. Rev. B 61, 846 (2000).

127 P. Chandra, P. Coleman, and A. I. Larkin, Phys. Rev. Lett. 64, 88 (1990).

128 C. Weber, L. Capriotti, G. Misguich, F. Becca, M. Elhajal, and F. Mila, Phys. Rev. Lett. 91, 177202 (2003).

129 R. Melzi, P. Carretta, A. Lascialfari, M. Mambrini, M. Troyer, P. Millet, and F. Mila, Phys. Rev. Lett. 85, 1318 (2000); R. Melzi, S. Aldrovandi, F. Tedoldi, P. Carretta, P. Millet, and F. Mila Phys. Rev. B 64, 24409 (2001).

130 P. Carretta, R. Melzi, N. Papinutto, and P. Millet, Phys. Rev. Lett. 88, 47601 (2002); P. Carretta, N. Papinutto, C. B. Azzoni, M. C. Mozzati, E. Pavarini, S. Gonthier, and P. Millet, Phys. Rev. B 66, 094420 (2002).

131 V. N. Kotov, J. Oitmaa, O. P. Sushkov, and W. H. Zheng, Phil. Mag. B 801483 (2000).

132 L. Capriotti, Int. J. Mod. Phys. B 15, 1799 (2001).

133 L. Capriotti, A. Fubini, T. Roscilde, and V. Tognetti, Phys. Rev. Lett. 92, 157202 (2004).

134 See the group homepage http://www.fi.infn.it/cmtg/. 